\title{
Órbitas Homoclínicas de Equações \\ Diferenciais Funcionais
}

\author{
Magda Kimico Kaibara
}

\author{
Orientação \\ Prof. Dr. José Gaspar. Ruas Filho
}

Dissertação apresentada ao Instituto de Ciências Matemáticas de São Carlos USP, como parte dos requisitos para obtenção do título de Mestre em Matemática.

\author{
USP - São Carlos \\ Maio 1992
}


À minha "Familia". 
Agradeço aos meus pais pela dedicação. Ao Gaspar pela paciência, atenção e incentivo. Aos amigos pelo coração sempre aberto. A Deus...

Este trabalho conta com apoio do CNPq e da Capes 


\begin{abstract}
Suppose an autonomous functional differential equation has an orbit $\Gamma$ which is homoclinic to a hyperbolic equilibrium point.

Our aim is to study a procedure for determining the behavior of solutions, close to $\Gamma$, of a functional differential equation which is a nonautonomous periodic perturbation of the original one.
\end{abstract}

This procedure uses exponential dichotomies and the Fredholm alternative. 


\section{Conteúdo}

Introdução

1 Preliminares 1

1.1 A Equação Diferẹncial Linear com Retardamento . . . . . . . 1

1.2 A Adjunta Formal e a Adjunta Verdadeira . . . . . . . . . . 9

2 Dicotomias Exponenciais 20

3 Alternativa de Fredhom para Soluções Limitadas de Equações Diferenciais Funcionais

4 Bifurcação de Órbitas Homoclínicas $\quad 54$

$\begin{array}{ll}\text { Bibliografia } & 62\end{array}$ 


\section{Introduçáo}

Suponhamos que uma equação diferencial funcional autônoma possui uma órbita $\Gamma$ homoclínica a um ponto de equilíbrio hiperbólico. Nosso objetivo é estudar um procedimento para determinar o comportamento das soluções perto de $\Gamma$ de uma equação que é uma perturbação não autônoma periódica da equação original.

Quando consideramos uma equação variacional linear perto de uma órbita homoclínica de uma equação autônoma num espaço de dimensão finita, Palmer [8] observou que o estudo do comportamento das soluções de sistemas periodicamente perturbados perto desta órbita pode ser estudado sob o ponto de vista das dicotomias exponencias. Explorando este fato e utilizando as técnicas de Liapunov-Schmidt e a Alternativa de Fredholm realizou este trabalho. A diferença entre o trabalho de Palmer e o nosso que é baseado em Lin [7] é o universo em que trabalhamos. Nós consideramos o espaço de Banach das funções contínuas num intervalo fechado com imagem em $\mathbf{R}^{\mathbf{n}}$ que denotamos por $C$.

Algumas definições e resultados básicos de equações diferenciais funcionais são apresentados no primeiro capítulo. Definimos o operador solução $T(t, s)$ do sistema

$$
\begin{aligned}
\dot{x}(t) & =L(t) x_{t}, \quad t \geq \sigma \\
x_{\sigma} & =\phi
\end{aligned}
$$

onde $L(t)$ pertence ao conjunto dos operadores lineares contínuos em $C$ tomando valores em $\mathrm{R}^{\mathrm{n}}$ e $L(t)$ é contínuo com respeito a $t$ num intervalo $J \subset \mathrm{R}$. Consideramos o sistema perturbado de $(0.1)$

$$
\begin{aligned}
\dot{x}(t) & =L(t) x_{t}+h(t), \quad t \geq \sigma \\
x_{\sigma} & =\phi
\end{aligned}
$$

cuja solução é dada pela Fórmula da Variação das Constantes. Estudamos uma - relação entre os operadores adjunto verdadeiro $T^{*}(s, t)$ e adjunto formal $\tilde{T}(s, t)$ associados a equação $(0.1)$ bem como uma relação entre as soluções do sistema 
linear não homogêneo (0.2) e as soluções do sistema adjunto formal

$$
y(s)+\int_{s}^{\infty} y(\alpha) \eta(\alpha, s-\alpha) d \alpha=\text { constante }
$$

No capítulo dois é definida dicotomia exponencial para o operador solução $T(t, s)$ da equação $(0.1)$ o que implicará na existência da dicotomia exponencial para o operador adjunto verdadeiro $T^{*}(s, t)$. Estudamos algumas propriedades das projeções associadas a essas dicotomias exponenciais. Alguns resultados básicos sobre perturbações de sistemas do tipo (0.1) são também apresentados. A parte central deste capítulo consiste no estudo da extensão do intervalo onde (0.1) possui dicotomia exponencial.

Apresentamos no terceiro capítulo teoremas que nos fornecem condições necessárias e suficientes para que uma função $x: \mathbf{R}_{+}\left(\mathbf{R}_{-}\right) \rightarrow \mathbf{R}^{\mathbf{n}}$ seja solução limitada da equação (0.2). Construímos um isomorfismo entre as soluções do sistema adjunto verdadeiro e as soluções do sistema adjunto formal da equação linear (0.1) que juntamente com os reșultados já obtidos garantem condições suficientes para que (0.1) possua dicotomia expoencial em $\mathbf{R}_{+}$e $\mathbf{R}_{-}$. Encerramos este capitulo com o teorema da. Alternativa de Fredholm para solucões limitadas da equação diferencial funcional (0.2). Além disso, são definidas fórmulas e significados para

(1) $T(t, s) X_{0}$

(2) $\int_{a}^{b} T(t, s) X_{0} h(s) d s$;

(3) $<\psi, T(t, s) X_{0}>=<T^{*}(s, t) \psi, X_{0}>$;

(4) $<\psi, \int_{a}^{b} T(t, s) X_{0} h(s) d s>=\int_{a}^{b}<\psi, T(t, s) X_{0}>h(s) d s$;

(5) $P(s) X_{0}, Q(s) X_{0}$;

(6) $T(t, s) P(s) X_{0}=P(t) T(t, s) X_{0}$;

(7) $<\psi, T(t, s) P(s) X_{0}>=<T^{*}(s, t) P^{*}(t) \psi, X_{0}>$;

$(8)<\psi, T(s, t) Q(t) X_{0}>=<T^{*}(t, s) Q^{*}(s) \psi, X_{0}>$

onde

$$
X_{0}(\theta)= \begin{cases}0 & -r \leq 0<0 \\ I & \theta=0 .\end{cases}
$$

Isto é necessário pois $X_{0}$ não é contínua e possui papel importante nas soluções do sitema linear não homogêneo. 
Finalizando, consideramos a equação escalar retardada:

$$
\dot{x}(t)=\alpha(x(t)) x(t)+\beta(x(t)) x(t-1)
$$

que supomos possuir uma trajetória homoclínica $p(t)$ a $x \equiv 0$. Estudamos então o comportamento das soluções perto de $p(t)$ do sistema periodicamente perturbado:

$$
\dot{x}(t)=\alpha(x(t)) x(t)+\beta(x(t)) x(t-1)+h\left(t, x_{t}, \mu\right)
$$

utilizando as técnicas de Liapunov-Schmidt e a Alternativa de Fredholm para soluções limitadas apresentada no capítulo três. 


\section{Capítulo 1}

\section{Preliminares}

Neste capítulo apresentamos resultados básicos que serão utilizados no desenvolvimento dos capítulos subsequentes. Na seção (1.1) definimos e apresentamos propriedades do operador solução da equação $\dot{x}(t)=L(t) x_{t}$ e a Fórmula da Variação das Constantes para a equação $\dot{x}(t)=L(t) x_{t}+h(t)$. Na seção (1.2) definimos os operadores adjunto fomal o adjunte verdadeire e resultadoc rela cionando estes operadores e as soluções do sistema linear não homogêneo com as soluções do sistema adjunto formal. A teoria apresentada neste capítulo pode ser encontrada em [6].

\subsection{A Equação Diferencial Linear com Retar- damento}

Para cada $(\sigma, \phi) \in \mathbf{R} \times C$, consideremos o sistema linear:

$$
\begin{aligned}
\dot{x}(t) & =L(t) x_{t}+h(t), \quad t \geq \sigma \\
x_{\sigma} & =\phi
\end{aligned}
$$

ou equivalentemente,

$$
\begin{aligned}
x(t) & =\phi(0)+\int_{\sigma}^{t} L(s) x_{s} d s+\int_{\sigma}^{t} h(s) d s, \quad t \geq \sigma \\
x_{\sigma} & =\phi
\end{aligned}
$$

onde: 
- $\phi$ pertence ao conjunto das funções contínuas em $[-r, 0]$ com valores em $\mathbf{R}^{n}$, isto é, a $C\left([-r, 0], \mathbf{R}^{\mathfrak{n}}\right)$ que denotaremos por $C$.

- $x_{t}(\theta) \stackrel{\text { def }}{=} x(t+\theta)$, onde $-r \leq \theta \leq 0$ e $x(t)$ está definida em algum intervalo $J \subset \mathbf{R}$ tomando valores em $\mathbf{R}^{\mathbf{n}}$.

- $h \in L_{l o c}^{1}\left([\sigma,+\infty), \mathbf{R}^{\mathbf{n}}\right)$, o espaço das funções definidas em $[\sigma,+\infty)$ com valores em $\mathbf{R}^{n}$, Lebesgue integráveis em cada compacto de $[\sigma,+\infty)$.

- $L(t)$ pertence ao conjunto dos operadores lineares contínuos em $C$ tomando valores em $\mathbf{R}^{\mathbf{n}}$, isto é, $L(t) \in \mathcal{L}\left(C, \mathbf{R}^{n}\right)$. Logo, existe uma função matricial $n \times n$ obtida do teorema de Riesz, $\eta(t, \theta)$, que é mensurável em $(t, \theta) \in \mathbf{R} \times \mathbf{R}$, normalizada tal que:

$$
\eta(t, \theta)= \begin{cases}0 & \theta \geq 0 \\ \eta(t,-r) & \theta \leq-r ;\end{cases}
$$

contínua à esquerda em $\theta \in\left(-r_{i} 0\right): \eta \underline{\eta}(t,$.$) possui variação limitada em$ $[-r, 0]$ para cada $t$, e existe uma função $m \in L_{l o c}^{1}((-\infty,+\infty), \mathbf{R})$ tal que:

$$
\begin{gathered}
L(t) \dot{\phi}=\int_{-r}^{0} d_{\theta} \eta(t, \theta) \phi(\theta) \\
|L(t) \phi| \leq m(t)|\phi|
\end{gathered}
$$

para todo $t \in(-\infty,+\infty)$ e $\phi \in C$. Claramente, $V_{\theta=-r}^{0} \eta(t, \theta) \leq m(t)$, onde $V_{\theta=-r}^{0} \eta(t, \theta)$ é a variação total de $\eta(t, \theta)$ em $[-r, 0]$.

Teorema 1.1 (Teorema de Arzelá - Áscoli) Seja $\mathcal{K}$ um espaço métrico compacto e $\mathcal{E} \subset C\left(\mathcal{K}, R^{n}\right)$, tal que:

(a) Existe um número real $N>0$ tal que $|f(x)| \leq N$ para todo $x \in \mathcal{K}$ e para todo $f \in \mathcal{E}$;

(b) $\mathcal{E}$ é equicontínuo.

Então $\mathcal{E}$ é relativamente compacto.

A demonstração deste teorema pode ser encontrada em [10]

Teorema 1.2 (Teorema não simétrico de Fubini) Seja $k(x)$ uma função de variação limitada sobre qualquer intervalo finito. Seja $p(x, u)$ Borel mensurável 
em $(x, u) ; p(x, u)$ de variação limitada em $u$ em qualquer intervalo finito para quase todo $x$ com respeito a $k(x)$. Denotemos por $V(x, u)$ a variação

$$
V(x, u)=\int_{0+}^{u+}\left|d_{v} p(x, v)\right| .
$$

Assumamos que $\int_{-\infty}^{+\infty} V(x, u)|d k(x)|$ existe (é finita) para todo u. Seja s $(u)$ Borel mensurável em $(-\infty,+\infty)$. Então a existência(finita) de

$$
\int_{-\infty}^{+\infty}|s(u)| d_{u} \int_{-\infty}^{+\infty} V(x, u)|d k(x)|
$$

implica a existência da

$$
\int_{-\infty}^{+\infty}|d k(x)| \int_{-\infty}^{+\infty}\left|s(u) \| d_{u} p(x, u)\right|
$$

a igualdade e reciprocamente. Além disso, temos:

$$
\int_{-\infty}^{+\infty} s(u) d_{u} \int_{-\infty}^{+\infty} p(x, u) d k(x)=\int_{-\infty}^{+\infty} d k(x) \int_{-\infty}^{+\infty} s(u) d_{u} p(x, u)
$$

A demonstração deste teorema pode ser encontrada em [3].

Consideremos , agora, o sistema linear:

$$
\begin{aligned}
\dot{x}(t) & =L(t) x_{t}, \quad t \geq \sigma \\
x_{\sigma} & =\phi
\end{aligned}
$$

com a hipótese adicional: $L(t)$ é contínua com respeito a $t \in J$ na norma de operadores.

Definição $1.1 O$ operador solução de (1.2) é a aplicação $T(t, s): C \rightarrow C$, definida por $T(t, s) \phi=x_{t}(s, \phi)$ que a cada $\phi \in C$, associa a solução $x_{t}(s, \phi)$ de (1.2) que no instante s possui a condição inicial $\phi$.

Lema 1.1 O operador solução possue as seguintes propriedades:

(i) $T(t, s)$ é um operador linear limitado;

(ii) a familia $\{T(t, s): t \geq s\} \subset \mathcal{L}(C)$ é uma familia de operadores de evolução, isto é;

(a) $T(s, s)=I$,

(b) $T(t, \tau) T(\tau, s)=T(t, s), t \geq \tau \geq s$;

(c) a aplicação $(t, s) \rightarrow T(t, s) \phi$ é contínua para cada $\phi$ em $C$ e $t \geq s$;

(iii) $T(t, s)$ é um operador compacto para $t \geq s+r$. 


\section{Demonstração}

(i) A linearidade de $T(t, s)$ segue da linearidade da $L(t)$ e da unicidade de soluçôes de (1.2).

Provemos a continuidade de $T(t, s)$.

A solução de (1.2) para cada $t, s$ fixados pode ser escrita como:

$$
T(t, s) \phi(\theta)= \begin{cases}\phi(\theta) & t+\theta \leq s \\ \phi(0)+\int_{s}^{t+\theta} L(u)(T(t, s) \phi) d u & t+\theta>s .\end{cases}
$$

Tomando-se a norma na equação acima e a seguir o sup em $\theta \in[-r, 0]$ obtemos:

$$
|T(t, s) \phi| \leq|\phi|+\int_{s}^{t}|L(u)||T(u, s) \phi| d u .
$$

Da continuidade de $L(u)$ em relação a $u$ pertencente ao compacto $[s, t]$ segue:

$$
|L(u)| \leq l .
$$

Então pela desigualdade de Gronwall:

$$
|T(t, s) \phi| \leq e^{l(t-s)}|\phi|
$$

para todo $t \geq s$.

(ii) $\mathrm{O}$ item (a) é imediato.

Provar que $T(t, \tau) T(\tau, s)=T(t, s)$, para todo $t \geq \tau \geq s$ é equivalente a provar que:

$$
x_{t}\left(\tau, x_{\tau}(s, \phi)\right)=x_{t}(s, \phi) .
$$

Em particular para $\tau=s$, o primeiro e o segundo membros da igualdade acima são iguais a $x_{t}(s, \phi)$. Então pela unicidade de soluções de (1.2) segue a igualdade para todo $t \geq \tau \geq s$.

(c) Notemos que:

$\left(a^{\prime}\right)$

$$
|T(t, s) \phi-\phi|=\sup _{\theta \in[-r, 0]}|T(t, s) \phi(\theta)-\phi(\theta)| \longrightarrow 0, \text { quando } t \rightarrow s .
$$

(b') Para $t \geq r \geq s$ :

$$
|T(t, r) \phi-T(t, s) \phi|=|T(t, r)(I-T(r, s)) \phi| \longrightarrow 0,
$$


quando $r \rightarrow s$, segue de (a) e da continuidade do operador solução.

(c') Para $t \geq w \geq r$ :

$$
|T(t, r) \phi-T(w, r) \phi|=|(T(t, w)-I) T(w, r) \phi| \longrightarrow 0
$$

quando $t \rightarrow w\left(\right.$ de $\left.\left(\mathrm{a}^{\prime}\right)\right)$. Consideremos agora $|T(t, s) \phi-T(w, r) \phi|$. Então ou $t \geq w$ ou $w \geq t$. Cada uma destas possibilidades divide-se em outras três, do seguinte modo:

(1) $t \geq w \geq r \geq s$ ou $t \geq w \geq s \geq r$ ou $t \geq s \geq w \geq r$;

(2) $w \geq t \geq s \geq r$ ou $w \geq t \geq r \geq s$ ou $w \geq r \geq t \geq s$.

No caso (1) temos:

$$
\begin{aligned}
|T(t, s) \phi-T(w, r) \phi| & =|T(t, s) \phi-T(t, r) \phi+T(t, r) \phi-T(w, r) \phi| \\
& \leq|T(t, s) \phi-T(t, r) \phi|+|T(t, r) \phi-T(w, r) \phi| \longrightarrow 0
\end{aligned}
$$

quando $(t, s) \rightarrow(w, r)$ para cada uma das possibilidades de (1) usando (b') e (c').

No caso (2) temos:

$$
|T(t, s) \phi-T(w, r) \phi ! \leq| T(t, s) \phi-T(w, s) \phi|+| T(w, s) \phi-T(\dot{w}, r) \phi \mid \longrightarrow 0 \text {, }
$$

quando $(t, s) \rightarrow(w, r)$ para cada uma das possibilidades de (2), usando (b') e (c').

Portanto, quando $(t, s) \rightarrow(w, r) ;|T(t, s) \phi-T(w, r) \phi| \rightarrow 0$.

(iii) Seja $S=\{\phi \in C:|\phi| \leq R\}$, onde $R>0$. C.

Mostremos que para cada $t, s$ fixados; $T(t, s) S$ é relativamente compacto em

A hipótese (a) do teorema de Arzelá - Áscoli segue da continuidade de $T(t, s)$. Verifiquemos (b).

Dado $\epsilon>0$, seja $\delta=\epsilon /\left(l R e^{l(t-s)}\right)$. Se $\left|\theta_{1}-\theta_{2}\right|<\delta$ entẫo:

$$
\begin{aligned}
\left|T(t, s) \phi\left(\theta_{2}\right)-T(t, s) \phi\left(\theta_{1}\right)\right| & =\left|x_{t}(s, \phi)\left(\theta_{2}\right)-x_{t}(s, \phi)\left(\theta_{1}\right)\right| \\
& =\left|x(s, \phi)\left(t+\theta_{2}\right)-x(s, \phi)\left(t+\theta_{1}\right)\right|
\end{aligned}
$$

Se $t-s \geq r$, o último membro é:

$$
\leq|\dot{x}(s, \phi)(\tilde{t})|\left|\theta_{2}-\theta_{1}\right|
$$


onde $\tilde{t} \in\left(t+\theta_{1}, t+\theta_{2}\right)$. Mas como,

$$
|\dot{x}(s, \phi)(\tilde{t})| \leq|L(\tilde{t})|\left|x_{\hat{t}}(s, \phi)\right| \leq l|T(\tilde{t}, s) \phi| \leq l e^{l(t-s)} R
$$

obtemos:

$$
\left|T(t, s) \phi\left(\theta_{2}\right)-T(t, s) \phi\left(\theta_{1}\right)\right| \leq \epsilon .
$$

Pelo Teorema de Áscoli, o fêcho de $T(t, s) S$ é compacto em $C$. Logo $T(t, s)$ é um operador compacto para $t-s \geq r$.

Teorema 1.3 Suponhamos que L satisfaz as hipóteses dadas inicialmente neste capítulo. Dados $\sigma \in \mathbf{R}, \phi \in C$ e $h \in L_{l o c}^{1}\left([\sigma,+\infty), \mathbf{R}^{\mathbf{n}}\right)$ existe uma única função $x(\sigma, \phi)$ definida e contínua em $[\sigma-r,+\infty)$ que satisfaz o sistema (1.1) em $[\sigma,+\infty)$.

Definição 1.2 Consideremos a função

$$
L: \mathbf{R} \times C \rightarrow \mathbf{R}^{\mathbf{n}}
$$

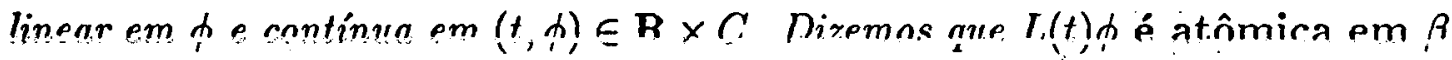
em $\mathbf{R} \times C$ se $\operatorname{det} A(t, \beta) \neq 0$, para todo $t \in \mathbf{R}$ onde

$$
A(t, \beta)=\eta\left(t, \beta^{+}\right)-\eta\left(t, \beta^{-}\right)
$$

Exemplo: Consideremos $\dot{x}(t)=x(t-1)$. Claramente, $L(t) \phi=\phi(-1)$ o que implica $\eta(t, \theta)=0$ se $\theta>-1$ e $\eta(t, \theta)=-1$ caso contrário. Consequentemente, $A(t,-1)=\eta\left(t,-1^{+}\right)-\eta\left(t,-1^{-}\right)=1$.

Lema 1.2 Consideremos o operador solução $T(t, s): C \rightarrow C$ da equação $\dot{x}(t)=$ $L(t) x_{t}$ onde $L: \mathbf{R} \times C \rightarrow \mathbf{R}^{\mathbf{n}}$ é contínua. Se $L(t) \phi$ é atômica em $-r$ em $\mathbf{R} \times C$ então $T(t, s)$ é injetora.

Teorema 1.4 (Fórmula da Variação das Constantes) Suponhamos que $L$ satisfaz as hipóteses anteriores, $h \in L_{\text {loc }}^{1}\left([\sigma,+\infty), \mathbf{R}^{n}\right)$ e $x(\sigma, \phi, h)$ é a solução do sistema (1.1), então:

$$
\begin{aligned}
x(\sigma, \phi, h)(t) & =x(\sigma, \phi, 0)(t)+\int_{\sigma}^{t} U(t, s) h(s) d s, t \geq \sigma \\
x_{\sigma} & =\phi
\end{aligned}
$$


onde $U(t, s)$ é a solução da equação:

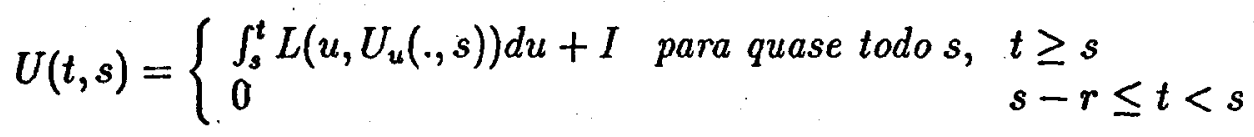

ou

$$
\begin{aligned}
\frac{\partial U(t, s)}{\partial t} & =L\left(t, U_{t}(., s)\right), t \geq s \text { para quase todo s e } t \\
U(t, s) & = \begin{cases}0 & s-r \leq t<s \\
I & t=s\end{cases}
\end{aligned}
$$

onde $U_{t}(., s)(\theta)=U(t+\theta, s),-r \leq \theta \leq 0$. A função $U(t, s)$ é chamada de matriz fundamental.

Observação 1.1 Podemos escrever a Fórmula da Variação das Constantes como:

$$
x_{t}(\sigma, \phi, h)=T(t, \sigma) \phi+\int_{\sigma}^{t} T(t, s) X_{0} h(s) d s, t \geq \sigma
$$

onde:

$$
\begin{aligned}
& T(t, s) X_{0}=U_{t}(., s) \\
& X_{0}(\theta)= \begin{cases}0 & -r \leq \theta<0 \\
I & \theta=0 .\end{cases}
\end{aligned}
$$

De fato, de (1.3) obtemos:

$$
\begin{aligned}
& x(\sigma, \phi, h)(t+\theta)=x(\sigma, \phi, 0)(t+\theta)+\int_{\sigma}^{t+\theta} U(t+\theta, s) h(s) d s, t+\theta \geq \sigma \\
& x(\sigma, \phi, h)(t+\theta)=\phi(t-\sigma+\theta) \quad \sigma-r \leq t+\theta \leq \sigma, \theta \in[-r, 0] .
\end{aligned}
$$

Como:

$$
\left\{\begin{aligned}
U(t+\theta, s) & =0 & & s>t+\theta \\
x(\sigma, \phi, 0)(t+\theta) & =\phi(t-\sigma+\theta) & & \sigma-r \leq t+\theta \leq \sigma
\end{aligned}\right.
$$

temos que:

$$
x(\sigma, \phi, h)(t+\theta)=x(\sigma, \phi, 0)(t+\theta)+\int_{\sigma}^{t} U(t+\theta, s) h(s) d s, t \geq \sigma, \theta \in[-r, 0]
$$

ou:

$$
x_{t}(\sigma, \phi, h)(\theta)=x_{t}(\sigma, \phi, 0)(\theta)+\int_{\sigma}^{t} U_{t}(., s)(\theta) h(s) d s, \quad t \geq \sigma, \theta \in[-r, 0]
$$


ou:

$$
x_{t}(\sigma, \phi, h)=x_{t}(\sigma, \phi, 0)+\int_{\sigma}^{t} U_{t}(., s) h(s) d s, t \geq \sigma
$$

onde a integral na expressão acima é uma integral no espaço euclidiano onde todas as operações usuais para integrais são válidas. Isto é, cada função é primeiro calculada em $\theta$ e então a integral é obtida como uma integral usual de $\mathbf{R}^{\mathbf{n}}$.

Como $U(t, s)$ é contínua em $t$ para $t \geq s$, segue da equação (1.4) que $U(t, s)$ possui a primeira derivada contínua em $t$ para $t>s+r$. Então, a equação (1.5) é satisfeita em $t$ para todo $t \geq s+r$ e para quase todo $s$. Portanto, podemos escrever:

$$
\begin{aligned}
& U_{t}(., s)=T(t, s) X_{0} \\
& X_{0}(\theta)= \begin{cases}0 & -r \leq \theta<0 \\
I & \theta=0\end{cases}
\end{aligned}
$$

Deste modo a Fórmula da Variação das Constantes torna-se:

$$
x_{t}(\sigma, \phi, h)=T(t, \sigma) \phi+\int_{\sigma}^{t} T(t, s) X_{0} h(s) d s, t \geq \sigma .
$$

Observação 1.2 Se para cada $t \geq \sigma, x_{t} \in C$ é dada por

$$
x_{t}=T(t, \sigma) \phi+\int_{o}^{t} T(t, s) X_{0} h(s) d s
$$

então a função $w:[\sigma-r,+\infty) \rightarrow \mathbf{R}$ definida por $w(t+\theta)=x_{t}(\theta), \theta \in[-r, 0]$, $t \geq \sigma$, satisfaz (1.1).

De fato,

$$
T(t, \sigma) \phi(\theta)=T(t+\theta, \sigma) \phi(0) \quad \text { para } \theta \in[-r, 0] \text { e } t \geq \sigma
$$

$\mathbf{e}$

$$
\int_{\sigma}^{t} T(t, s) X_{0}(\theta) h(s) d s=\left\{\begin{array}{lr}
\int_{\sigma}^{t+\theta} T(t+\theta, s) X_{0}(0) h(s) d s & t+\theta \geq \sigma \\
0 & t+\theta \leq \sigma .
\end{array}\right.
$$

Portanto, se definirmos:

$$
\begin{aligned}
w(t) & =x_{t}(0) & & t \geq \sigma \\
w(\sigma+\theta) & =\phi(\theta) & & \theta \in[-r, 0]
\end{aligned}
$$

claramente obtemos: $x_{t}(\theta)=w(t+\theta), \theta \in[-r, 0], t \geq \sigma$.

Portanto, $x(\sigma, \phi, h)$ é solução se, e somente se, satisfaz

$$
x_{t}=T(t, \sigma) \phi+\int_{\sigma}^{t} T(t, s) X_{0} h(s) d s .
$$




\subsection{A Adjunta Formal e a Adjunta Verdadeira}

Definição $1.3 \cdot$ A equação:

$$
y(s)+\int_{s}^{\infty} y(\alpha) \eta(\alpha, s-\alpha) d \alpha=c t e
$$

onde $y \in \mathbf{R}^{\mathbf{n} *}$, espaço dos vetores linha de dimensão $n$, é chamada equação adjunta formal de (1.2).

Exemplo: Se $\dot{x}(t)=A(t) x(t)+B(t) x(t-1)$ então $y(s)+\int_{s}^{s+1}[-(y \alpha)] A(\alpha) d \alpha+$ $\int_{s+1}^{t}[-y(\alpha)][A(\alpha)+B(\alpha)] d \alpha=$ cte é a sua equção adjunta formal:

No estudo que se segue, frequentemente será conveniente usar uma função $p$ que definimos como $p(t, s)=\eta(t, s-t)$. Então:

$$
p(t, s)=\left\{\begin{array}{lc}
0 & s \geq t \\
p(t, t-r) & s \leq t-r
\end{array}\right.
$$

com isto a equação adjunta formal torna-se

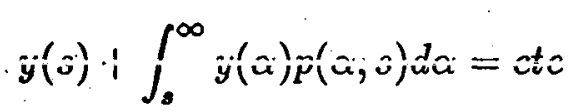

Seja $B_{0}=B_{0}[-r, 0]$, o espaço de Banach das funções $\psi:[-r, 0] \rightarrow \mathbf{R}^{\mathbf{n} *}$ de variação limitada em $[-r, 0]$; contínuas à esquerda em $(-r, 0), \psi(0)=0$ e cuja norma é dada por $|\psi|=V_{\theta=-r}^{0} \psi$.

Teorema 1.5 Para cada $t \in \mathbf{R}$ e $\psi \in B_{0}$, existe uma única $y: \mathbf{R} \rightarrow \mathbf{R}^{\mathbf{n} *}$ que se anula em $[t,+\infty)$, satisfaz a equação(1.6) em $(-\infty, t-r]$, e tal que $y_{t}=\psi$. Para cada.s $\leq t$, seja $y_{s}$ definido por:

$$
\begin{aligned}
& y_{s}(\theta)=y(s+\theta), \quad-r \leq \theta<0 \\
& y_{s}(0)=0 .
\end{aligned}
$$

Então esta solução pode ser escrita para cada $s \leq t$ como:

$$
y_{s}=\tilde{T}(s, t) y_{t}=\tilde{T}(s, t) \psi
$$

onde $\tilde{T}(s, t): B_{0} \rightarrow B_{0}$ é linear e limitado.

Lema $1.3\{\tilde{T}(s, t): s \leq t\}$ satisfaz:

(i) $\tilde{T}(t, t)=I$

(ii) $\tilde{T}(s, \tau) \tilde{T}(\tau, t)=\tilde{T}(s, t), s \leq \tau \leq t$. 
Demonstração Análoga ao item (ii) do lema 1.1.

Teorema 1.6 Se $x$ satisfaz a equação (1.1) em $[\sigma,+\infty)$, então para todo $t \geq \sigma$,

$$
x(t)=Y(\sigma, t) x(\sigma)+\int_{\sigma-\boldsymbol{r}}^{\sigma^{-}} d_{\beta}\left\{\int_{\sigma}^{t} Y(\alpha, t) p(\alpha, \beta) d \alpha\right\} x(\beta)+\int_{\sigma}^{t} Y(\alpha, t) h(\alpha) d \alpha
$$

onde a função matricial $n \times n, Y$,é definida por:

$$
Y(\sigma, t)= \begin{cases}0 & \sigma>t \\ I-\int_{\sigma}^{t} Y(\alpha, t) p(\alpha, \sigma) d \alpha & \sigma \leq t\end{cases}
$$

Além disso, $Y(\sigma, t)$ é absolutamente contínua em $t$ em cada conjunto compacto de $t \leq \sigma$, localmente de variação limitada em $\sigma$, e $Y(\sigma, t)=U(t, \sigma)$ para quase todo $\sigma$, onde $U(t, \sigma)$ é definida pela equação (1.4).

Consideremos, agora, $C^{*}$, o dual de $C$. Se $\psi \in C^{*}$, o funcional linear $\phi \mapsto \psi(T(t, s) \phi)$ é a composição de duas aplicações lineares contínuas, $\psi$ e $T(t, s)$, e portanto é um elemento de $C^{*}$. Denotamos este funcional por $T^{*}(s, t) \psi$ para

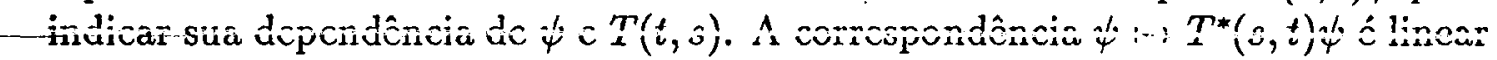
e define o operador $T^{*}(s, t)$ de $C^{*}$ em $C^{*}$. Temos então a definição:

Definição 1.4 $O$ operador $\mathbf{T}^{*}(\mathrm{~s}, \mathrm{t})$ é chamado o adjunto (verdadeiro) de $T(t, s)$ $e$ é definido por:

$$
<T^{*}(s, t) \psi, \phi>=<\psi, T(t, s) \phi>.
$$

Identificaremos $B_{0}$ com o espaço dual de $\mathrm{C}, \mathrm{C}^{*}$, usando a relação:

$$
\langle\psi, \phi\rangle=\int_{-r}^{0} d \psi(\theta) \phi(\theta)
$$

para todo $\psi \in B_{0}$ e $\phi \in C$, ainda quando a função $\phi$ possua uma quantidade enumerável de pontos de descontinuidade.

Lema 1.4 O operador adjunto possui as seguintes propriedades:

(i) A familia $\left\{T^{*}(s . t): s \leq t\right.$ em J\} satisfaz:

(a) $T^{*}(t, t)=I$,

(b) $T^{*}(s, \tau) T^{*}(\tau, t)=T^{*}(s, t), t \geq \tau \geq s$;

(ii) $s \rightarrow<T^{*}(s, t) \psi, \phi>$ é continua com respeito a $s \leq t$ em $J, \psi \in C^{*}$, $\phi \in C$. 


\section{Demonstração}

(i) (a) Como $<T^{*}(t, t) \psi, \phi>=<\psi, T(t, t) \phi>=<\psi, \phi>$, para todo $\psi \in C^{*}$, para todo $\phi \in C$, segue que $T^{*}(t, t)=I$.

(b) Seja $s \leq \tau \leq t$. Então:

$$
\begin{aligned}
\left\langle T^{*}(s, \tau) T^{*}(\tau, t) \psi, \phi>\right. & =\left\langle T^{*}(\tau, t) \psi, T(\tau, s) \phi>\right. \\
& =\langle\psi, T(t, \tau) T(\tau, s) \phi> \\
& =<\psi, T(t, s) \phi> \\
& =<T^{*}(s, t) \psi, \phi>
\end{aligned}
$$

para todo $\psi \in C^{*}$ e para todo $\phi \in C$. Assim,

$$
T^{*}(s, \tau) T^{*}(\tau, t)=T^{*}(s, t)
$$

para todo $s \leq \tau \leq t$ em $J$

(ii) Seja $\tau, s \leq t, \psi \in C^{*}$ e $\phi \in C$. Quando $\tau \rightarrow s$,

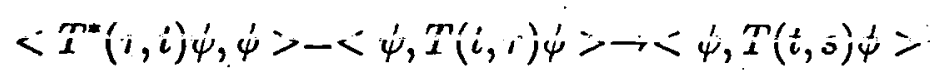

pela continuidade forte de $T(t, s)$ em $(t, s)$. Mas,

$$
<\psi, T(t, s) \phi>=<T^{*}(s, t) \psi, \phi>
$$

logo,

$$
<T^{*}(\tau, t) \psi, \phi>\rightarrow<T^{*}(s, t) \psi, \phi>. \square
$$

Definição 1.5 Um operador $\Omega$ em $B_{0}$ é dito quase- nilpotente se o zero é o único elemento no espectro de $\Omega$.

Se $\Omega$ é o operador de Volterra,

$$
\hat{\Omega} \psi(\theta)=\int_{\theta}^{0} \psi(\alpha) \mu(\alpha, \theta) d \alpha,-r \leq \theta \leq 0
$$

onde $\mu(\alpha, \theta)$ é uma função matricial $n \times n$, que satisfaz as mesmas condições que $\eta$, então $\Omega$ é quase-nilpotente.

Observação 1.3 Todo operador nilpotente é quase-nilpotente. A recíproca não é verdadeira (ver [10]). 
Teorema 1.7 Para cada $t \geq \sigma$,

$$
T^{*}(\sigma, t)=(I+\Omega(\sigma)) \tilde{T}(\sigma, t)(I+\Omega(t))^{-1}
$$

onde $\tilde{T}(\sigma, t)$ é dado no teorema 1.5 e $\Omega(\sigma)$ é o operador quase-nilpotente em $B_{0}$ definido por

$$
(\Omega(\sigma) \psi)(\theta)=\int_{\theta}^{0} \psi(\alpha) \eta(\sigma+\alpha, \theta-\alpha) d \alpha,-r \leq \theta \leq 0
$$

para todo $\sigma \in \mathbf{R}$ e $\psi \in B_{0}$.

Demonstração Para cada $\phi \in \mathbf{C}$ e $\psi \in B_{0}$ seja $x=x(\sigma, \phi, 0)$ tal que $x_{\sigma}=\phi$, $x_{t}=T(t, \sigma) \phi$ para $t \geq \sigma$. Estendemos $\psi$ definindo:

$$
\psi(\xi)= \begin{cases}\psi(-r) & \xi \leq-r \\ 0 & \xi \geq 0\end{cases}
$$

e estendemos $x$ de modo que seja contínua em $(-\infty,+\infty)$. Assim:

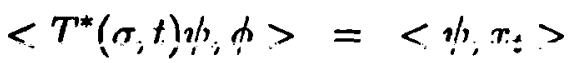

$$
\begin{aligned}
& =\int_{-\infty}^{0}[d \psi(\theta)] x(t+\theta) \\
& =\int_{-\infty}^{\sigma-t-r}[d \psi(\theta)] x(t+\theta)+\int_{\sigma-t-r}^{(\sigma-t)^{-}}[d \psi(\theta)] x(t+\theta) \\
& +\int_{(\sigma-t)-}^{\sigma-t}[d \psi(\theta)] x(t+\theta)+\int_{\sigma-t}^{0}[d \psi(\theta)] x(t+\theta) \text {. }
\end{aligned}
$$

Como para $\theta \in(-\infty, \sigma-t-r], \psi(\theta)=\psi(-r)$ a primeira integral é igual a zero, bem como a terceira, desde que $\psi$ é contínua à esquerda em $(-r, 0)$. E do teorema 1.6 obtemos que:

$$
\begin{aligned}
<T^{*}(\sigma, t) \psi, \phi>= & \int_{-r}^{0^{-}}\left[d_{\xi} \psi(\xi+\sigma-t)\right] \phi(\xi) \\
& +\int_{\sigma-t}^{0}[d \psi(\theta)] Y(\sigma, t+\theta) \phi(0) \\
& +\int_{\sigma-t}^{0}[d \psi(\theta)] \int_{\sigma-r}^{\sigma^{-}} d_{\beta}\left\{\int_{\sigma}^{t+\theta} Y(\alpha, t+\theta) p(\alpha, \beta) d \alpha\right\} x((\beta) 10)
\end{aligned}
$$

Fazendo uma mudança de variáveis na última integral, $(\beta=\sigma+\xi)$,temos que:

$$
\int_{\sigma-t}^{0}[d \psi(\theta)] \int_{-r}^{0^{-}} d_{\xi}\left\{\int_{\sigma}^{t+\theta} Y(\alpha, t+\theta) p(\alpha, \sigma+\xi) d \alpha\right\} \phi(\xi) .
$$


Como $Y(\alpha, t+\theta)=0$ para $\alpha>t+\theta$, a expressão acima torna-se:

$$
\int_{\sigma-t}^{0}[d \psi(\theta)] \int_{-r}^{0^{-}} d_{\xi}\left\{\int_{\sigma}^{t} Y(\alpha, t+\theta) p(\alpha, \sigma+\xi) d \alpha\right\} \phi(\xi) .
$$

Pelo teorema de Fubini não simétrico trocamos a ordem de integração

$$
\begin{aligned}
& \int_{-r}^{0^{-}} d_{\xi}\left\{\int_{\sigma-t}^{0}[d \psi(\theta)] \int_{\sigma}^{t} Y(\alpha, t+\theta) p(\alpha, \sigma+\xi) d \alpha\right\} \phi(\xi) \\
= & \int_{-r}^{0^{-}} d_{\xi}\left\{\int_{\sigma}^{t}\left[\int_{\sigma-t}^{0}[d \psi(\theta)] Y(\alpha, t+\theta)\right] p(\alpha, \sigma+\xi) d \alpha\right\} \phi(\xi) \\
= & \left.\int_{-r}^{0^{-}} d_{\xi}\left\{\int_{\sigma}^{t}\left[\int_{\alpha-t}^{0} d \psi(\theta)\right] Y(\alpha, t+\theta)\right] p(\alpha, \sigma+\xi) d \alpha\right\} \phi(\xi) .
\end{aligned}
$$

A última igualdade verifica-se, já que, $Y(\alpha, t+\theta)=0$ para $\alpha>t+\theta$.

Definimos:

$$
y(\alpha, t)= \begin{cases}0 & \alpha>t \\ \int_{\alpha-t}^{0}[d \psi(\theta)] Y(\alpha, t+\theta) & \alpha \leq t\end{cases}
$$

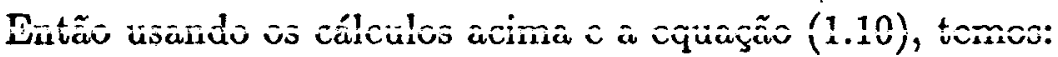

$$
\begin{aligned}
\left\langle T^{*}(\sigma, t) \psi, \phi>=\right. & \int_{-r}^{0^{-}} d_{\xi}\left[\psi(\xi+\sigma-t)+\int_{\sigma}^{t} y(\alpha, t) p(\alpha, \sigma+\xi) d \alpha\right] \phi(\xi) \\
& +y(\sigma, t) \phi(0) .
\end{aligned}
$$

Como a igualdade acima deve valer para todo $\phi \in C$, desta expressão e da definição do produto obtemos que $\left[T^{*}(\sigma, t) \psi\right](\xi)$ possui uma descontinuidade em $\theta=0$ e, em particular que

$$
\left[T^{*}(\sigma, t) \psi\right]\left(0^{-}\right)=-y(\sigma, t)
$$

Provemos, agora, que $y(\sigma, t)$ é solução da equação adjunta formal.

Temos que:

$$
\begin{aligned}
y(\sigma, t) & =\int_{\sigma-t}^{0}[d \psi(\theta)] Y(\sigma, t+\theta) \\
& =\int_{\sigma-t}^{0}[d \psi(\theta)]-\int_{\sigma-t}^{0}[d \psi(\theta)] \int_{\sigma}^{t+\theta} Y(\alpha, t+\theta) p(\alpha, \sigma) d \alpha
\end{aligned}
$$

pois

$$
Y(\sigma, t)= \begin{cases}0 & \sigma>t \\ I-\int_{\sigma}^{t} Y(\alpha, t) p(\alpha, \sigma) d \alpha & \sigma \leq t\end{cases}
$$


Como $Y(\sigma, t)=0$ para $\sigma>t$

$$
\int_{\sigma}^{t+\theta} Y(\alpha, t+\theta) p(\alpha, \sigma) d \alpha=\int_{\sigma}^{t} Y(\alpha, t+\theta) p(\alpha, \sigma) d \alpha .
$$

Logo,

$$
y(\sigma, t)=-\psi(\sigma-t)-\int_{\sigma-t}^{0}[d \psi(\theta)]\left(\int_{\sigma}^{t} Y(\alpha, t+\theta) p(\alpha, \sigma) d \alpha\right)
$$

Usando o teorema de Fubini não simétrico no último termo obtemos,

$$
y(\sigma, t)=-\psi(\sigma-t)-\int_{\sigma}^{t}\left\{\int_{\sigma-t}^{0}[d \psi(\theta)] Y(\alpha, t+\theta)\right\} p(\alpha, \sigma) d \alpha .
$$

Como $Y(\sigma, t)=0$ para $\sigma>t$

$$
\begin{aligned}
y(\sigma, t) & =-\psi(\sigma-t)-\int_{\sigma}^{t}\left\{\int_{\alpha-t}^{0}[d \psi(\theta)] Y(\alpha, t+\theta)\right\} p(\alpha, \sigma) d \alpha \\
& =-\psi(\sigma-t)-\int_{\sigma}^{t} y(\alpha, t) p(\alpha, \sigma) d \alpha .
\end{aligned}
$$

Portanto,

$$
y(\sigma, t)+\int_{\sigma}^{t} y(\alpha, t) p(\alpha, \sigma) d \alpha=-\psi(\sigma-t), \quad \sigma \leq t .
$$

Como $\psi(\sigma-t)=\psi(-r)$ para $\sigma \leq t-r$, segue que

$$
y(\sigma, t)+\int_{\sigma}^{t} y(\alpha, t) p(\alpha, \sigma) d \alpha=-\psi(-r), \sigma \leq t-r
$$

e $y(\sigma, t)$ como uma função de $\sigma$ é uma solução da equação adjunta formal (1.6). Do teorema 1.5, podemos portanto escrever

$$
y_{\sigma}(., t)=\tilde{T}(\sigma, t) y_{t}(., t) .
$$

A condição inicial $y_{t}(., t)$ é dada pela equação (1.13) por:

$$
y(t+\theta, t)+\int_{t+\theta}^{t} y(\alpha, t) p(\alpha, t+\theta) d \alpha=-\psi(\theta),-r \leq \theta \leq 0
$$

ou

$$
y(t+\theta, t)+\int_{\theta}^{0} y(t+\alpha, t) \eta(t+\alpha, \theta-\alpha) d \alpha=-\psi(\theta), \quad-r \leq \theta \leq 0 .
$$

Comparando com a equação (1.9), temos $[I+\Omega(t)] y_{t}(., t)=-\psi \mathrm{e}$

$$
y_{\sigma}(., t)=-\tilde{T}(\sigma, t)(I+\Omega(t))^{-1} \psi, \sigma \leq t .
$$


Voltando em (1.11), usando a equação (1.13) e a condição (1.12), para $-r \leq \xi$ $<0$,

$$
\begin{aligned}
{\left[T^{*}(\sigma, t) \psi\right](\xi)=} & \psi(\xi+\sigma-t)+\int_{\sigma}^{t} y(\alpha, t) p(\alpha, \sigma+\xi) d \alpha \\
= & -y(\sigma+\xi, t)-\int_{\sigma+\xi}^{t} y(\alpha, t) p(\alpha, \sigma+\xi) d \alpha \\
& +\int_{\sigma}^{t} y(\alpha, t) p(\alpha, \sigma+\xi) d \alpha \\
= & -y(\sigma+\xi, t)-\int_{\sigma+\xi}^{\sigma} y(\alpha, t) p(\alpha, \sigma+\xi) d \alpha \\
& -\int_{\sigma}^{t} y(\alpha, t) p(\alpha, \sigma+\xi) d \alpha+\int_{\sigma}^{t} y(\alpha, t) p(\alpha, \sigma+\xi) d \alpha \\
= & -y(\sigma+\xi, t)-\int_{\xi}^{0} y(\sigma+\beta, t) \eta(\sigma+\beta, \xi-\beta) d \beta \\
= & -\left[(I+\Omega(\sigma)) y_{\sigma}(., t)\right](\xi) .
\end{aligned}
$$

Usando a expressão (1.14) na equação acima, obtemos:

$$
T^{*}(\sigma, t)=(I+\Omega(\sigma)) \tilde{T}(\sigma, t)(I+\Omega(t))^{-1}, \quad \sigma \leq t . \square
$$

Lema 1.5 Seja $-\infty<a<b<+\infty \operatorname{com}|a-b|>M$, para todo $M>0$.

Se $x$ é uma solução de (1.1) em $t \in[a,+\infty) \operatorname{com} x(t)=\phi(t)$ para $a-r \leq t \leq a$ onde $\phi \in C$ e se y é uma solução da equação adjunta formal para $s \in(-\infty, b]$ e $y(\alpha)=\psi(\alpha)$ para $\alpha \in[b, b+r]$ onde $\psi \in B_{0}$ então para todo $\sigma, t$ tal que $a \leq \sigma<t \leq b$ temos

$$
\begin{aligned}
y(t) x(t)+ & \int_{t-r}^{t} d_{s}\left[\int_{t}^{t+r} y(\alpha) p(\alpha, s) d \alpha\right] x(s) \\
= & y(\sigma) x(\sigma)+\int_{\sigma-r}^{\sigma} d_{s}\left[\int_{\sigma}^{\sigma+r} y(\alpha) p(\alpha, s) d \alpha\right] x(s) \\
& +\int_{\sigma}^{t} y(\alpha) h(\alpha) d \alpha, \sigma \leq t
\end{aligned}
$$

Demonstração O sistema (1.1) pode ser escrito como

$$
\dot{x}(t)=\int_{-r}^{0} d_{\theta} \eta(t, \theta) x(t+\theta)+h(t)
$$

ou

$$
\dot{x}(t)=\int_{t-r}^{t} d_{s} p(t, s) x(s)+h(t) .
$$


Seja $a<\sigma<t<b$. Assumimos que $\sigma+r<t$. Posteriormente esta condição será eliminada.

Como $x(t)$ é uma solução de (1.15) em $[a, b]$ que satisfaz $x(t)=\phi(t)$ em $[a-r, a]$ onde $\phi \in C ; x(t)$ é uma função absolutamente contínua em $[a, b]$, satisfazendo (1.15) em algum intervalo $[a, t]$ e com condição inicial $\phi \in C$ em $[a-r, a], \log o$, $x(t)$ é de variação limitada em $[a, b]$. Então usando a fórmula de integração por partes obtemos:

$$
\int_{\sigma}^{t} d y(\alpha) x(\alpha)+\int_{\sigma}^{t} y(\alpha) \dot{x}(\alpha) d \alpha=\left.y(\alpha) x(\alpha)\right|_{\sigma} ^{t}
$$

ou

$$
\begin{aligned}
y(t) x(t)-y(\sigma) x(\sigma)= & \int_{\sigma}^{t} d y(\alpha) x(\alpha)+\int_{\sigma}^{t} y(\alpha)\left\{\int_{\alpha-r}^{\alpha} d_{s} p(\alpha, s) x(s)+h(\alpha)\right\} d \alpha \\
= & \int_{\sigma}^{t} d y(\alpha) x(\alpha)+\int_{\sigma}^{t} y(\alpha) h(\alpha) d \alpha \\
& +\int_{\sigma}^{t} y(\alpha)\left\{\int_{\alpha-r}^{\alpha} d_{s} p(\alpha, s) x(s)\right\} d \alpha .
\end{aligned}
$$

Consid̉eremos o úitimo termo do segundio menìro da equação acinia. Essie termo pode ser escrito como:

$$
\int_{\sigma}^{t} y(\alpha)\left\{\int_{\sigma-r}^{t} d_{s} p(\alpha, s) x(s)\right\} d \alpha
$$

pois $p(\alpha, s)=c t e=0$ para $s \geq \alpha$ e $p(\alpha, s)=c t e=p(\alpha, \alpha-r)$ para $s \leq \alpha-r$. Usando o teorema de Fubini não simétrico obtemos:

$$
\int_{\sigma}^{t} y(\alpha)\left\{\int_{\sigma-r}^{t} d_{s} p(\alpha, s) x(s)\right\} d \alpha=\int_{\sigma-r}^{t} d_{s}\left\{\int_{\sigma}^{t} y(\alpha) p(\alpha, s) d \alpha\right\} x(s) .
$$

Usando que $\sigma+r<t$ e $p(\alpha, s)=0$ para $s \geq \alpha$, o último termo pode ser escrito como:

$$
\begin{aligned}
& \int_{\sigma-r}^{\sigma^{-}} d_{s}\left[\int_{\sigma}^{t} y(\alpha) p(\alpha, s) d \alpha\right] x(s) \\
+ & \int_{\sigma^{-}}^{\sigma} d_{s}\left[\int_{\sigma}^{t} y(\alpha) p(\alpha, s) d \alpha\right] x(s) \\
+ & \int_{\sigma}^{t-r} d_{s}\left[\int_{s}^{t} y(\alpha) p(\alpha, s) d \alpha\right] x(s) \\
+ & \int_{t-r}^{t-} d_{s}\left[\int_{s}^{t} y(\alpha) p(\alpha, s) d \alpha\right] x(s) \\
+ & \int_{t^{-}}^{t} d_{s}\left[\int_{s}^{t} y(\alpha) p(\alpha, s) d \alpha\right] x(s)
\end{aligned}
$$


Como para $s \in[\sigma-r, \sigma]$ o termo

$$
\int_{\sigma+r}^{t} y(\alpha) p(\alpha, s) d \alpha
$$

é uma função constante de $s$, e como $y ; p(\alpha, s)$ são contínuas a esquerda obtemos:

$$
\begin{aligned}
& \int_{\sigma-r}^{\sigma^{-}} d_{s}\left[\int_{\sigma}^{\sigma+r} y(\alpha) p(\alpha, s) d \alpha\right] x(s) \\
+ & \int_{\sigma}^{t-r} d_{s}\left[\int_{s}^{t} y(\alpha) p(\alpha, s) d \alpha\right] x(s) \\
+ & \int_{t-r}^{t-} d_{s}\left[\int_{s}^{t} y(\alpha) p(\alpha, s) d \alpha\right] x(s) .
\end{aligned}
$$

Somando e subtraindo o termo:

$$
\int_{t-r}^{t^{-}} d_{s}\left[\int_{t}^{t+r} y(\alpha) p(\alpha, s) d \alpha\right] x(s)
$$

na expressão acima obtemos:

$$
\begin{aligned}
& \int_{\sigma-r}^{\sigma^{-}} d_{s}\left[\int_{\sigma}^{\sigma+r} y(\alpha) p(\alpha, s) d \alpha\right] x(s) \\
+ & \int_{\sigma}^{t-r} d_{s}\left[\int_{s}^{t} y(\alpha) p(\alpha, s) d \alpha\right] x(s) \\
- & \int_{t-r}^{t-} d_{s}\left[\int_{t}^{t+r} y(\alpha) p(\alpha, s) d \alpha\right] x(s) \\
+ & \int_{t-r}^{t-} d_{s}\left[\int_{0}^{t+r} y(\alpha) p(\alpha, s) d \alpha\right] x(s)
\end{aligned}
$$

que pode. ser escrito

$$
\begin{aligned}
& \int_{\sigma-r}^{\sigma^{-}} d_{s}\left[\int_{\sigma}^{\sigma+r} y(\alpha) p(\alpha, s) d \alpha\right] x(s) \\
+ & \int_{\sigma}^{t-r} d_{s}\left[\int_{s}^{t+r} y(\alpha) p(\alpha, s) d \alpha\right] x(s) \\
- & \int_{t-r}^{t^{-}} d_{s}\left[\int_{t}^{t+r} y(\alpha) p(\alpha, s) d \alpha\right] x(s) \\
+ & \int_{t-r}^{t^{-}} d_{s}\left[\int_{s}^{t+r} y(\alpha) p(\alpha, s) d \alpha\right] x(s)
\end{aligned}
$$

pois para $s \in[\sigma, t-r]$ o termo

$$
\int_{t}^{t+r} \cdot y(\alpha) p(\alpha, s) d \alpha
$$


é constante em $s$.

Combinando dois termos da expressão acima obtemos:

$$
\begin{aligned}
& \int_{\sigma-r}^{\sigma^{-}} d_{s}\left[\int_{\sigma}^{\sigma+r} y(\alpha) p(\alpha, s) d \alpha\right] x(s) \\
+ & \int_{\sigma}^{t^{-}} d_{s}\left[\int_{s}^{t+r} y(\alpha) p(\alpha, s) d \alpha\right] x(s) \\
- & \int_{t-r}^{t^{-}} d_{s}\left[\int_{t}^{t+r} y(\alpha) p(\alpha, s) d \alpha\right] x(s) .
\end{aligned}
$$

Substituindo esta expressão no último termo da equação (1.16) obtemos:

$$
\begin{aligned}
y(t) x(t)-y(\sigma) x(\sigma)= & \int_{\sigma}^{t} d y(\alpha) x(\alpha)+\int_{\sigma}^{t} y(\alpha) h(\alpha) d \alpha \\
& +\int_{\sigma}^{t} d_{s}\left[\int_{s}^{t+r} y(\alpha) p(\alpha, s) d \alpha\right] x(s) \\
& +\int_{\sigma-r}^{\sigma^{-}} d_{s}\left[\int_{\sigma}^{\sigma+r} y(\alpha) p(\alpha, s) d \alpha\right] x(s) \\
& -\int_{t-r}^{t^{-}} d_{s}\left[\int_{t}^{t+r} y(\alpha) p(\alpha, s) d \alpha\right] x(s)
\end{aligned}
$$

Agora para $s \in[\sigma, t]$ temos que

$$
\int_{t+r}^{\infty} y(\alpha) p(\alpha, s) d \dot{\alpha}
$$

é constante em $s$. Assim o terceiro termo do segundo membro da equação (1.17) pode ser escrito como

$$
\int_{\sigma}^{t} d_{s}\left[\int_{s}^{\infty} y(\alpha) p(\alpha, s) d \alpha\right] x(s)
$$

Então a equação (1.17) tornar-se-á

$$
\begin{aligned}
y(t) x(t)+ & \int_{t-r}^{t^{-}} d_{s}\left[\int_{t}^{t+r} y(\alpha) p(\alpha, s) d \alpha\right] x(s) \\
= & y(\sigma) x(\sigma)+\int_{\sigma-r}^{\sigma^{-}} d_{s}\left[\int_{\sigma}^{\sigma+r} y(\alpha) p(\alpha, s) d \alpha\right] x(s) \\
& +\int_{\sigma}^{t} d_{s}\left[y(s)+\int_{s}^{\infty} y(\alpha) p(\alpha, s) d \alpha\right] x(s) \\
& +\int_{\sigma}^{t} y(\alpha) h(\alpha) d \alpha
\end{aligned}
$$


Como $y$ é solução da equação adjunta formal, obtemos:

$$
\begin{aligned}
y(t) x(t)+ & \int_{t-r}^{t^{-}} d_{s}\left[\int_{t}^{t+r} y(\alpha) p(\alpha, s) d \alpha\right] x(s) \\
= & y(\sigma) x(\sigma)+\int_{\sigma-r}^{\sigma^{-}} d_{s}\left[\int_{\sigma}^{\sigma+r} y(\alpha) p(\alpha, s) d \alpha\right] x(s) \\
& +\int_{\sigma}^{t} y(\alpha) h(\alpha) d \alpha
\end{aligned}
$$

Se definirmos $\mathcal{B}_{t}(y, x)$ como

$$
\mathcal{B}_{t}(y, x)=y(t) x(t)+\int_{t-r}^{t^{-}} d_{s}\left[\int_{t}^{t+r} y(\alpha) p(\alpha, s) d \alpha\right] x(s)
$$

então (1.18) tornar-se-á

$$
\mathcal{B}_{\sigma}(y, x)+\int_{\sigma}^{t} y(\alpha) h(\alpha) d \alpha=\mathcal{B}_{t}(y, x)
$$

quando $\sigma<t$ e $\sigma+r<t$.

É fácil ver que se $F(\sigma)=F(t)$ parà $\sigma$ e $t$ arbitrários com $\sigma<t$ e $\sigma+r<t$, então $F(\sigma)=F(t)$ para $\sigma$ e $t$ arbitrários, $\sigma<t$. Isto implica que $F$ é uma função constante. Assim, ao longo das soluções $x$ e $y$ a função $\mathcal{B}_{t}(x, y)$ definida em (1.19) é uma constante em $t, a<t<b$. Deste modo a igualdade (1.18) é válida para todo $\sigma<t$ arbitrários em $[a, b]$. 


\section{Capitulo 2}

\section{Dicotomias Exponenciais}

Neste capítulo apresentamos a definição de dicotomia exponencial e alguns lemas, dentre os quais ressaltamos aqueles referentes à extensão do intervalo onde a equação $\dot{x}(t)=L(t) x_{t}$ possui uma dicotomia exponencial (lemas $2.6,2.7 \mathrm{e}$ 2.8). Notamos que uma condição necessária para que a dicotomia exponencial

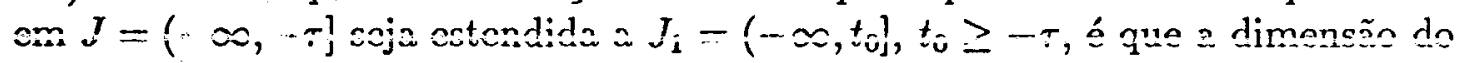
subespaço instável $\mathcal{R} Q(t)$ para $t \in J$ seja igual a dimensão do subespaço instável para $t \in J_{1}$. Provaremos que esta também é uma condição suficiente.

Necessitamos das seguintes definições:

Definição 2.1 Definimos os anuladores $E^{0} e^{0} \mathrm{~F}$ como:

$$
\begin{aligned}
& E^{0}=\left\{x^{*} \in X^{*}:<x^{*}, x>=0, \forall x \in E\right\} \\
& { }^{0} F=\left\{x \in X:<x^{*}, x>=0, \forall x^{*} \in F\right\}
\end{aligned}
$$

onde $X$ é um espaço de Banach, $X^{*}$ seu espaço dual, $E \subset X$ e $F \subset X^{*}$.

Definição $2.2 O$ operador solução $T(t, s)$ possui uma dicotomia exponencial em um intervalo $J$ com constantes $K$ e $\alpha$ positivas se existem projeções contínuas $P(s), Q(s)=I-P(s)$ fortemente continuas em s tal que:

(i) $T(t, s) P(s)=P(t) T(t, s), t \geq s$ em $J$;

(ii) $\left.T(t, s)\right|_{\mathcal{R} Q(s)}, t \geq s$ é um isomorfismo de $\mathcal{R} Q(s)$ sobre $\mathcal{R} Q(t)$ e a inversa de $\left.T(t, s)\right|_{\mathcal{R} Q(s)}$ é definida como $T(s, t): \mathcal{R} Q(t) \rightarrow \mathcal{R} Q(s)$;

(iii) $|T(t, s) P(s)| \leq K e^{-\alpha(t-s)}, t \geq s$ em ; 
(iv) $|T(s, t) Q(t)| \leq K e^{-\alpha(t-s)}, t \geq s$ em $J$.

$\mathcal{R} P(s)$ e $\mathcal{R} Q(s), s \in J$, são denominados de subespaços estável e instável respectivamente.

Observação 2.1 Segue diretamente de (i) que $\mathcal{R} P(t)$ e $\mathcal{R} Q(t)$ são "invariantes" sob $T(t, s)$.

Observação 2.2 Como $T(t, s)$ é compacto para $t-s \geq r$ e $\mathcal{R} Q(t)$ é completo (pois é fechado em $C$ ), $\mathcal{R} Q(t)$ possui dimensão finita (ver [10, pág. 298]).

Lema 2.1 Suponhamos que $T(t, s)$ possui dicotomia exponencial em $J$ e que $T(t, s) \phi_{s}=\phi_{t}$ onde $\phi_{t} \in \mathcal{R} P(t)$. Então $\phi_{s} \in \mathcal{R} P(s)$.

Demonstração Suponhamos $\phi_{s}=\phi_{s}^{P}+\phi_{s}^{Q}$ onde $\phi_{s}^{P} \in \mathcal{R} P(s)$ e $\phi_{s}^{Q} \in \mathcal{R} Q(s)$. Logo,

$$
\phi_{t}=T(t, s) \phi_{s}=T(t, s) \phi_{s}^{P}+T(t, s) \phi_{s}^{Q}
$$

com $T(t, s) \phi_{s}^{P} \in \mathcal{R} P(t)$ e $T(t, s) \phi_{s}^{Q} \in \mathcal{R} Q(t)$ visto que $\mathcal{R} P(s)$ e $\mathcal{R} Q(s)$ são invariantes. Portanto,

$$
T(t, s) \phi_{s}^{P}-\phi_{t}=T(t, s) \phi_{s}^{Q} \in \mathcal{R} Q(t) \cap \mathcal{R} P(t)
$$

daí, $T(t, s) \phi_{s}^{Q}=0$ o que implica $\phi_{s}^{Q}=0$, já que, $\left.T(t, s)\right|_{\mathcal{R} Q(s)}$ é um isomorfismo. Deste modo, temos que $\phi_{s} \in \mathcal{R} P(s)$.

Teorema 2.1 Suponhamos que a equação (1.2) tem dicotomia exponencial com projeções $P(t)$ e $Q(t)$ em R. Então:

$$
\mathcal{R} P(s)=\{\phi \in C: T(t, s) \phi \text { é limitada para } t \geq s\}
$$

$e$

$\mathcal{R} Q(t)=\{\phi \in C: T(s, t) \phi$ está definida para todo $s \leq t$ e é limitada para $s \leq t\}$.

Demonstração Mostremos que

$$
\mathcal{R} P(s)=\{\phi \in C: T(t, s) \phi \dot{e} \text { limitada para } t \geq s\} .
$$


Suponhamos que $\phi \in \mathcal{R} P(s)$. Então

$$
|T(t, s) \phi|=|T(t, s) P(s) \phi| \leq K e^{-\alpha(t-s)}|\phi|
$$

e $T(t, s) \phi$ é limitada para $t \geq s$.

Suponhamos agora que $\phi \notin \mathcal{R} P(s)$, então

$$
T(t, s) \phi=T(t, s) P(s) \phi+T(t, s) Q(s) \phi .
$$

e $T(t, s) P(s) \phi$ é limitada para $t \geq s$. Ainda:

$$
|Q(s) \phi|=|T(s, t) Q(t) T(t, s) Q(s) \phi| \leq K e^{-\alpha(t-s)}|T(t, s) Q(s) \phi| .
$$

Daí,

$$
|T(t, s) Q(s) \phi| \geq \frac{1}{K} e^{\alpha(t-s)}|Q(s) \phi| \longrightarrow+\infty
$$

quando $t \rightarrow+\infty$. Portanto,

$$
\mathcal{R} P(s)=\{\phi \in C: T(t, s) \phi \text { é limitada para } t \geq s\} .
$$

Provemos que:

$\mathcal{R} Q(t)=\{\phi \in C: T(s, t) \phi$ está definida para todo $s \leq t$ e é limitada para $s \leq t\}$.

É imediato que se $\phi \in \mathcal{R} Q(t)$ então $T(s, t) \phi$ está definida e é limitada para $s \leq t$.

Suponhamos que $\phi \notin \mathcal{R} Q(t)$, então tomando

$$
\begin{aligned}
& \phi^{P}=P(t) \phi \\
& \phi^{Q}=Q(t) \phi
\end{aligned}
$$

temos que se $T(s, t) \phi$ está definida para $s \leq t$ então, como $T(s, t) \phi^{Q}$ está definida para $s \leq t$ devemos ter $T(s, t) \phi^{P}$ definida para $s \leq t$. Como $T(s, t) \phi^{Q}$ é limitada para $s \leq t$, devemos mostrar que $T(s, t) \phi^{P}$ não é limitada para $s \leq t$. De fato:

$$
|P(t) \phi|=|T(t, s) P(s) T(s, t) P(t) \phi| \leq K e^{-\alpha(t-s)}|T(s, t) P(t) \phi|
$$

$\mathbf{e}$

$$
|T(s, t) P(t) \phi| \geq \frac{1}{K} e^{\alpha(t-s)}|P(t) \phi| \longrightarrow+\infty
$$

quando $s \rightarrow-\infty$

Deste modo, $T(s, t) \phi$ não é limitado para $s \leq t$ e portanto segue que: $\mathcal{R} Q(t)=\{\phi \in C: T(s, t) \phi$ está definida para todo $s \leq t$ e é limitada para $s \leq t\} . \square$ 
Lema 2.2 $S e P^{*}(s)$ éo adjunto de $P(s)$ então $\left(P^{*}(s)\right)^{2}=P^{*}(s)$ e $P^{*}(s)$ é weak contínua em $s \in J$.

Demonstração Usando que $P^{2}(s)=P(s)$ obtemos:

$$
\left\langle\left(P^{*}(s)\right)^{2} \psi, \phi\right\rangle=\langle\psi, P(s) \phi\rangle=\left\langle P^{*}(s) \psi, \phi\right\rangle
$$

para todo $\psi \in C^{*}$ e para todo $\phi \in C$. Logo, $\left(P^{*}(s)\right)^{2}=P^{*}(s)$.

Seja, agora, $t \in J$, tal que $t \rightarrow s$, assim,

$$
\left\langle P^{*}(t) \psi, \phi\right\rangle=\langle\psi, P(t) \phi\rangle \longrightarrow\langle\psi, P(s) \phi\rangle=\left\langle P^{*}(s) \psi, \phi\right\rangle
$$

segue da continuidade forte de $P$ em $s$. Portanto,

$$
\left\langle P^{*}(t) \psi, \phi>\longrightarrow<P^{*}(s) \psi, \phi>\right.\text {. }
$$

Lema $2.3 \mathcal{R} Q^{*}(s)$ é isomorfo a $(\mathcal{R} Q(s))^{*}$.

Demonstração Sabendo que $(\mathcal{R} P(s))^{0}=\mathcal{R} Q^{*}(s)$ definimos a aplicação:

$$
\begin{aligned}
f: \mathcal{R} Q^{*}(s) & \rightarrow(\mathcal{R} Q(S))^{*} \\
\psi & \mapsto f(\psi): \mathcal{R} Q(s) \rightarrow \mathbf{R} \\
& f(\psi)(\phi)=<\psi, \phi>.
\end{aligned}
$$

Claramente, $f$ está bem definida e é linear.

Suponhamos que $0 \neq \psi \in \mathcal{R} Q^{*}(s), f(\psi) \phi=\langle\psi, \phi\rangle=0$. Sabemos que para todo $\phi \in C, \phi=\phi_{1}+\phi_{2}$ onde $\phi_{1} \in \mathcal{R} P(s)$ e $\phi_{2} \in \mathcal{R} Q(s)$, logo,

$$
0=\langle\psi, \phi\rangle=\left\langle\psi, \phi_{1}\right\rangle+\left\langle\psi, \phi_{2}\right\rangle=\left\langle\psi, \phi_{2}\right\rangle,
$$

o que implica, $\psi=0$. Contradição! Portanto, $f(\psi) \neq 0$ e $f$ é injetora.

Além disso, dado $\xi \in(\mathcal{R} Q(s))^{*}$, existe $\psi \in \mathcal{R} Q^{*}(s), \psi=\xi$, tal que $f(\psi)=\xi$. Mostremos que $\psi \in \mathcal{R} Q^{*}(s)$. De fato, como $\xi \in(\mathcal{R} Q(s))^{*}$ :

$$
<\xi, \phi>=<\xi, Q(s) \phi>=\left\langle Q^{*}(s) \xi, \phi>\right.
$$

para todo $\phi \in \mathcal{R} Q(s)$, dai, $Q^{*}(s) \xi=\xi$, o que implica $\xi \in \mathcal{R} Q^{*}(s)$.

Portanto, $\operatorname{dim}\left(\mathcal{R} Q^{*}(s)\right)=\operatorname{dim}(\mathcal{R} Q(s))^{*}$. 
Lema 2.4 $O$ operador adjunto $T^{*}(s, t)$ tem uma dicotomia exponencial em $J$ com projeções $P^{*}(s)$ e $Q^{*}(s)$, exceto que a continuidade forte é substituída pela continuidade weak*, ou seja;

(i) $T^{*}(s, t) P^{*}(t)=P^{*}(s) T^{*}(s, t), s \leq t$, em $J$

(ii)' $\left.T^{*}(s, t)\right|_{\mathcal{R} Q^{*}(t)}, s \leq t$, é um isomorfismo de $\mathcal{R} Q^{*}(t)$ sobre $\mathcal{R} Q^{*}(s)$ e $T^{*}(t, s): \mathcal{R} Q^{*}(s) \rightarrow \mathcal{R} Q^{*}(t)$ é definido como a inversa de $\left.T^{*}(s, t)\right|_{\mathcal{R} Q^{*}(t)}$ (ou a adjunta de $T(s, t): \mathcal{R} Q(t) \rightarrow \mathcal{R} Q(s))$;

(iii)' $\left|T^{*}(s, t) P^{*}(t)\right| \leq K e^{-\alpha(t-s)}, s \leq t$, em $J$;

(iv)' $\left|T^{*}(t, s) Q^{*}(s)\right| \leq K e^{-\alpha(t-s)}, s \leq t$, em $J$.

\section{Demonstração}

(i)' Segue da definição de $T^{*}(s, t), P^{*}(s)$ e do item (i) da definição de dicotomia exponencial.

(ii)' Primeiramente mostraremos que $\left.T^{*}(s, t)\right|_{\mathcal{R} Q^{*}(t)}$ é injetora.

Seja $\xi \in \mathcal{R} Q^{*}(t)$ tal que $T^{\prime *}(s, t) \xi=U$. Se $x=x_{1}+x_{2}$ com $x_{1} \in \mathcal{K} Q(t)$ e $x_{2} \in \mathcal{R} P(t)$ temos:

$\left\langle\xi, x>=\left\langle Q^{*}(t) \xi, x_{1}+x_{2}\right\rangle=<\xi, Q(t)\left(x_{1}+x_{2}\right)>=<\xi, Q(t) x_{1}\right\rangle=\left\langle\xi, x_{1}>\right.$.

Da hipótese de dicotomia exponencial (ii) existe $y_{1} \in \mathcal{R} Q(s)$ tal que $T(t, s) y_{1}=$ $x_{1}$, assim

$$
<\xi, x_{1}>=<\xi, T(t, s) y_{1}>=<T^{*}(s, t) \xi, y_{1}>=0 .
$$

Obtemos: $\langle\xi, x\rangle=\left\langle\xi, x_{1}\right\rangle=0$ para todo $x$.

Assim, $\xi=0$ e $\left.T^{*}(s, t)\right|_{R Q^{*}(t)}$ é $1-1$.

Se $\eta \in \mathcal{R} Q^{*}(s)$, seja $\xi$ definido por:

$$
<\xi, x>=<\eta, y_{1}>
$$

onde $y_{1} \in \mathcal{R} Q(s)$ é tal que $T(t, s) y_{1}=x_{1}$ com $x=x_{1}+x_{2}, x_{1} \in \mathcal{R} Q(t)$ e $x_{2} \in \mathcal{R} P(t)$.

Da definição (ii) de dicotomia exponencial segue que $\xi$ está bem definida. Também, $\xi \in \mathcal{R} Q^{*}(t)$ desde que para $x \in X$,

$$
\left\langle P^{*}(t) \xi, x>=<\xi, P(t) x>=0 .\right.
$$


Para provar que $T^{*}(s, t) \xi=\eta$ seja $y=y_{1}+y_{2} \in C$ com $y_{1} \in \mathcal{R} Q(s)$ e $y_{2} \in \mathcal{R} P(s)$, então:

$$
<T^{*}(s, t) \xi, y>=<\eta, y_{1}>+<\eta, y_{2}>=<\eta, y>.
$$

portanto, $T^{*}(s, t) \xi=\eta$.

(iii)' Temos:

$$
\begin{aligned}
\left\langle(T(t, s) P(s))^{*} \psi, \phi>\right. & =\langle\psi, T(t, s) P(s) \phi\rangle \\
& =\left\langle T^{*}(s, t) \psi, P(s) \phi\right\rangle \\
& =\left\langle P^{*}(s) T^{*}(s, t) \psi, \phi\right\rangle
\end{aligned}
$$

$\log 0$

$$
(T(t, s) P(s))^{*}=P^{*}(s) T^{*}(s, t)
$$

De (i)' temos que

$$
\left|T^{*}(s, t) P^{*}(t)\right|=\left|P^{*}(s) T^{*}(s, t)\right|
$$

e como

$$
\left|P^{*}(s) T^{*}(s, t)\right|=\left|(T(t, s) P(s))^{*}\right|=|T(t, s) P(s)|
$$

segue da hipótese de dicotomia exponencial (iii) que

$$
\left|T^{*}(s, t) P^{*}(t)\right| \leq K e^{-\alpha(t-s)} .
$$

(iv)' Temos:

$$
T^{*}(s, t) Q^{*}(t)=Q^{*}(s) T^{*}(s, t) .
$$

Como $\left.T^{*}(s, t)\right|_{\mathcal{R} Q^{*}(t)}$ admite inversa $T^{*}(t, s)$, aplicando-a primeiro à esquerda $\mathrm{e}$ depois à direita na igualdade anterior obtemos:

$$
Q^{*}(t) T^{*}(t, s)=T^{*}(t, s) Q^{*}(s)
$$

além disso:

$$
\begin{aligned}
\left\langle(T(s, t) Q(t))^{*} \psi, \phi>\right. & =\langle\psi, T(s, t) Q(t) \phi> \\
& =\left\langle T^{*}(t, s) \psi, Q(t) \phi>\right. \\
& =\left\langle Q^{*}(t) T^{*}(t, s) \psi, \phi>\right.
\end{aligned}
$$

para todo $\phi$. Logo, $(T(s, t) Q(t))^{*}=Q^{*}(t) T^{*}(t, s)$.

Portanto, $\left|T^{*}(t, s) Q^{*}(s)\right|=\left|Q^{*}(t) T^{*}(t, s)\right|=\left|(T(s, t) Q(t))^{*}\right|=|T(s, t) Q(t)| \leq K e^{-\alpha(t-s)}$ 
Teorema 2.2 Suponhamos que o operador adjunto de $T(t, s)$, isto é, $T^{*}(s, t)$ tem dicotomia exponencial em $\mathbf{R}$ com projeções $P^{*}(t)$ e $Q^{*}(t)$. Então:

$$
\mathcal{R} P^{*}(t)=\left\{\psi \in C^{*}: T^{*}(s, t) \psi \text { é limitada para } s \leq t\right\}
$$

e

$$
\mathcal{R} Q^{*}(s)=\left\{\psi \in C^{*}: T^{*}(t, s) \psi \text { está definida para } s \leq t \text { e é limitada para } s \leq t\right\}
$$

Demonstração Análoga ao teorema 2.1.

Utilizaremos o seguinte resultado:

Lema 2.5 Seja $J=\mathbf{R}_{+}\left(\mathbf{R}_{-}\right.$ou $\left.\mathbf{R}\right)$ : Se (1.2) tem uma dicotomia exponencial em $J$ com projeção $P(t), B(t)$ é um operador linear continuo para $t \in J$, contínua com respeito a $t$ na norma de operadores e $|B(t)| \leq \delta$ para todo $t \in J$, então:

$$
\dot{x}(t)=(L(t)+B(t)) x_{t}
$$

tem uma äicotomia exponenciai em $j$ com projeçäo $\tilde{F}(i), \tilde{F}(i) \rightarrow F(i j)$ quando $t \rightarrow+\infty(-\infty$ ou $\pm \infty)$ na norma de operadores se $\delta$ é suficientemente pequeno.

Para maiores informações veja [4] e [5].

Lema 2.6 Seja $J=(-\infty,-\tau]$ e suponhamos (1.2) possui uma dicotomia exponencial em $J$. Seja $t_{0}>-\tau$ e $T\left(t_{0},-\tau\right) \phi \neq 0$ para todo $0 \neq \phi \in \mathcal{R} Q(-\tau)$. Então (1.2) possui uma dicotomia exponencial em $\left(-\infty, t_{0}\right]$ com as projeções $\tilde{P}(t), \tilde{Q}(t) \rightarrow P(t), Q(t)$ exponencialmente quando $t \rightarrow-\infty$ na norma de operadores.

\section{Denonstração Seja}

$$
X_{1}(t)=\left\{\begin{array}{lr}
\mathcal{R} Q(t) & \text { para } t \leq-\tau \\
\{T(t,-\tau) \phi: \phi \in \mathcal{R} Q(-\tau)\} & \text { para }-\tau \leq t \leq t_{0}
\end{array}\right.
$$

Suponhamos $\operatorname{dim} \mathcal{R} Q(t)=m$ para $t \leq-\tau$. Em particular, $\operatorname{dim} \mathcal{R} Q(-\tau)=m$.

Seja $B=\left\{\phi_{1}, \phi_{2}, \ldots, \phi_{m}\right\}$ base de $\mathcal{R} Q(-\tau)$. 
Tomemos $B_{\phi}=\left\{T(t,-\tau) \phi_{1}, T(t,-\tau) \phi_{2}, \ldots, T(t,-\tau) \phi_{m}\right\}$.

Claramente $X_{1}(t)=\left[B_{\phi}\right]$ para $t \in\left[-\tau, t_{0}\right]$.

Seja,

$$
\beta_{1} T(t,-\tau) \phi_{1}+\beta_{2} T(t,-\tau)+\cdots+\beta_{m} T(t,-\tau) \phi_{m}=0
$$

logo,

$$
T(t,-\tau)\left(\beta_{1} \phi_{1}+\beta_{2} \phi_{2}+\cdots+\beta_{m} \phi_{m}\right)=0
$$

então,

$$
T\left(t_{0}, t\right) T(t,-\tau)\left(\beta_{1} \dot{\phi}_{1}+\beta_{2} \phi_{2}+\cdots+\beta_{m} \phi_{m}\right)=0
$$

ou seja,

$$
T\left(t_{0},-\tau\right)\left(\beta_{1} \phi_{1}+\beta_{2} \phi_{2}+\cdots+\beta_{m} \phi_{m}\right)=0
$$

mas, $\beta_{1} \phi_{1}+\beta_{2} \phi_{2}+\cdots+\beta_{m} \phi_{m} \in \mathcal{R} Q(-\tau)$, logo, devemos ter :

$$
\beta_{1} \phi_{1}+\beta_{2} \phi_{2}+\cdots+\beta_{m} \phi_{m}=0
$$

Como $B$ é base, $\beta_{1}=\beta_{2}=\cdots=\beta_{m}=0$, portanto, $B_{\phi}$ é linearmente independente e $\operatorname{dim} X_{1}(t)=m$ para todo $t \leq t_{0}$, o que implica $X_{1}(t)$ é fechado em $C$

Como $X_{1}(-\tau)$ é invariante sob $T(t,-\tau)$ (pela definição de $X_{1}(t)$ para $t \in$ $\left.\left[-\tau, t_{0}\right]\right)$ e como acabamos de provar $T(t,-\tau) \phi=0$, para todo $\phi \in \mathcal{R} Q(-\tau)$ implica $\phi=0$ temos que $\left.T(t,-\tau)\right|_{X(-\tau)}$ é um isomorfismo de $X_{1}(-\tau)$ sobre $X_{1}(t)$. Em particular quando $t \in\left[t_{0},-\tau\right]$ e $s \in(-\infty,-\tau], T(t, s)=T(t,-\tau) T(-\tau, s)$ e quando $t, s \in\left[t_{0},-\tau\right]$ com $t \geq s, T(t, s)=T(t,-\tau) T(-\tau, s)$ onde neste caso $T(-\tau, s)$ é a inversa de $T(s,-\tau)$. Logo, para todo $t, s$ em $\left(-\infty, t_{0}\right] ;\left.T(t, s)\right|_{X_{1}(s)}$ é um isomorfismo de $X_{1}(s)$ sobre $X_{1}(t)$.

Seja $X_{2}\left(t_{0}\right)$ subespaço fechado de $C$ complementar de $X_{1}\left(t_{0}\right)$, isto é

$$
C=X_{1}\left(t_{0}\right) \oplus X_{2}\left(t_{0}\right) .
$$

Seja,

$$
X_{2}(t)=\left\{\phi: T\left(t_{0}, t\right) \phi \in X_{2}\left(t_{0}\right)\right\}, \quad t \leq t_{0} .
$$

Segue diretamente do fato que $X_{2}\left(t_{0}\right)$ é um subespaço fechado de $C$ e da continuidade de $T\left(t_{0}, t\right)$ que $X_{2}(t)$ é um subespaço fechado de $C$. Além disso:

$$
C=X_{1}(t) \oplus X_{2}(t), \quad t \leq t_{0}
$$


De fato,

(i) Seja $\phi \in X_{1}(t) \cap X_{2}(t)$. Como $\phi \in X_{1}(t)$, do isomorfismo $\left.T\left(t_{0}, t\right)\right|_{X_{1}(t)}$, $T\left(t_{0}, t\right) \phi \in X_{1}\left(t_{0}\right)$. Mas $\phi \in X_{2}(t)$ o que implica da definição de $X_{2}(t)$ que $T\left(t_{0}, t\right) \phi \in X_{2}\left(t_{0}\right)$. Portanto, $T\left(t_{0}, t\right) \phi \in X_{1}\left(t_{0}\right) \cap X_{2}\left(t_{0}\right)$. Logo, $T\left(t_{0}, t\right) \phi=0 \mathrm{e}$ então $\phi=0$.

(ii) Seja $\phi \in C$. Temos então que:

$$
T\left(t_{0}, t\right) \phi=\phi_{1}+\phi_{2}
$$

onde $\phi_{1} \in X_{1}\left(t_{0}\right)$ e $\phi_{2} \in X_{2}\left(t_{0}\right)$. Já que, $\phi_{1} \in X_{1}\left(t_{0}\right)$ existe $\tilde{\phi}_{1} \in X_{1}(t)$ tal que $T\left(t_{0}, t\right) \tilde{\phi}_{1}=\phi_{1}$. Logo,

$$
T\left(t_{0}, t\right)\left(\phi-\tilde{\phi}_{1}\right)=\phi_{2} \in X_{2}\left(t_{0}\right)
$$

o que implica, da definição de $X_{2}(t)$, que $\phi-\tilde{\phi}_{1} \in X_{2}(t)$. Portanto,

$$
\phi=\tilde{\phi}_{1}+\left(\phi-\tilde{\phi}_{1}\right) \in X_{1}(t)+X_{2}(t)
$$

Definimos então $\tilde{P}(t), \tilde{Q}(t)$ projeções em $C$ tal que

$$
\begin{aligned}
& \mathcal{R} \tilde{Q}(t)=X_{1}(t) \\
& \mathcal{R} \tilde{P}(t)=X_{2}(t)
\end{aligned}
$$

para $t \leq t_{0}$. Devido ao isomorfismo $\left.T(t, s)\right|_{X_{1}(s)}: X_{1}(s) \rightarrow X_{1}(t), \mathcal{R} \tilde{Q}(s)$ é invariante sob $T(t, s)$ para $t_{0} \geq t \geq s$. Além disso:

$$
T\left(t_{0}, t\right)\left[T(t, s) X_{2}(s)\right]=T\left(t_{0}, s\right) X_{2}(s)
$$

para $t_{0} \geq t \geq s$. Da definição de $X_{2}(s), T\left(t_{0}, s\right) X_{2}(s) \subset X_{2}\left(t_{0}\right)$,isto é,

$$
T\left(t_{0}, t\right)\left[T(t, s) X_{2}(s)\right] \subset X_{2}\left(t_{0}\right)
$$

o que implica,

$$
T(t, s) X_{2}(s) \subset X_{2}(t)
$$

donde obtemos, $\mathcal{R} \tilde{P}(s)$ é invariante sob $T(t, s)$. Logo, para todo $\phi=\phi_{1}+\phi_{2}$ onde $\phi_{1} \in \mathcal{R} \tilde{Q}(s)$ e $\phi_{2} \in \mathcal{R} \tilde{P}(s)$ obtemos que

$$
\begin{aligned}
T(t, s) \tilde{P}(s) \phi & =\tilde{P}(t) T(t, s) \phi, \\
T(t, s) \tilde{Q}(s) \phi & =\tilde{Q}(t) T(t, s) \phi
\end{aligned}
$$


$t \geq s \operatorname{em}\left(-\infty, t_{0}\right]$

Da última igualdade obtemos:

$$
\tilde{Q}(t)=T\left(t, t_{0}\right) T\left(t_{0}, t\right) \tilde{Q}(\hat{\imath})=T\left(t, t_{0}\right) \tilde{Q}\left(t_{0}\right) T\left(t_{0}, t\right), \quad t \leq t_{0}
$$

$\mathrm{E}$ como $(t, s) \mapsto T(t, s) \phi$ é contínua para todo $\phi \in C, t \mapsto \tilde{Q}(t) \phi$ é contínua, do Princípio da Limitação Uniforme obtemos que $\tilde{Q}(t)$ é limitado em norma para $t$ num subconjunto compacto de $\left(-\infty, t_{0}\right]$. Cono $\tilde{P}(t)=I-\tilde{Q}(t)$ temos também que $t \mapsto \tilde{P}(t) \phi$ é contínua e $\tilde{P}(t)$ é limitado em norma para $t$ num subconjunto compacto de $\left(-\infty, t_{0}\right]$.

Visto que $\mathcal{R} \tilde{Q}(s)=\mathcal{R} Q(s)$ implica $Q(s) \tilde{Q}(s)=\tilde{Q}(s)$ e $\tilde{Q}(s) Q(s)=Q(s)$ para $s \leq-\tau$ temos:

$$
\begin{aligned}
\tilde{Q}(s) & =\tilde{Q}(s)(Q(s)+P(s)) \\
& =Q(s)+\tilde{Q}(s) P(s) \\
& =Q(s)+T(s,-\tau) \tilde{Q}(-\tau) T(-\tau, s) P(s) \\
& =Q(s)+T(s,-\tau) Q(-\tau) \tilde{Q}(-\tau) T(-\tau, s) P(s)
\end{aligned}
$$

logo,

$$
\tilde{Q}(s)-Q(s)=T(s,-\tau) Q(-\tau) \tilde{Q}(-\tau) T(-\tau, s) P(s)
$$

e das hipóteses (iii) e (iv) de dicotomia obtemos:

$$
\begin{aligned}
|\tilde{Q}(s)-Q(s)| & \leq k e^{\alpha(\tau+s)} M k e^{\alpha(\tau+s)} \\
& \leq k^{2} M e^{2 \alpha(\tau+s)}
\end{aligned}
$$

assim,

$$
\begin{aligned}
|\tilde{Q}(s)| & \leq|\tilde{Q}(s)-Q(s)|+|Q(s)| \\
& \leq k^{2} M e^{2 \alpha(\tau+s)}+|Q(s)|
\end{aligned}
$$

e, portanto, $|\tilde{Q}(s)| \rightarrow|Q(s)|$ exponencialmente em norma quando $s \rightarrow-\infty$. Fazendo $t=s$ em $|T(s, t) Q(t)| \leq k e^{-\alpha(t-s)}$ obtemos $|Q(s)| \leq k$. Como $P(s)=I-$ $Q(s),|\tilde{P}(s)| \rightarrow|P(s)|$ exponencialmente em norma quando $s \rightarrow-\infty$ e $|P(s)| \leq k$. Além disso, no compacto $\left[-\tau, t_{0}\right], \tilde{Q}(s)$ e $\tilde{P}(s)$ são limitadas. Daí, $\tilde{Q}(s)$ e $\tilde{P}(s)$ são uniformemente limitadas em norma para $s \in\left(-\infty, t_{0}\right]$. Digamos: $|\tilde{P}(s)|,|\tilde{Q}(s)| \leq$ $k_{1}$ para $s \leq t_{0}$.

Para provarmos as estimativas dicotômicas devemos analisar três casos:

Caso 1: $-\tau \geq t \geq s$ 
Temos:

$$
\begin{aligned}
|T(s, t) \tilde{Q}(t)| & =\left|T^{\prime}(s, t) Q(t) \tilde{Q}(t)\right| \\
& =|T(s, t) Q(t)||\tilde{Q}(t)| \\
& \leq k k_{1} e^{-\alpha(t-s)}
\end{aligned}
$$

Como $\tilde{Q}(t) Q(t)=Q(t)$ segue que:

$$
\tilde{P}(t)=\tilde{P}(t)(P(t)+Q(t))=\tilde{P}(t) P(t)+\tilde{P}(t) \tilde{Q}(t) Q(t)=\tilde{P}(t) P(t)
$$

portanto,

$$
\begin{aligned}
|T(t, s) \tilde{P}(s)| & =|\tilde{P}(t) T(t, s)|=|\tilde{P}(t) P(t) T(t, s)| \\
& \leq|\tilde{P}(t) \| T(t, s) P(s)| \\
& \leq k k_{1} e^{-\alpha(t-s)}
\end{aligned}
$$

Mostramos até agora que (1.2) tem dicotomia exponencial relativamente as projeções $\tilde{P}(t)$ e $\tilde{Q}(t)$ em $(-\infty,-\tau]$.

Caso 2: $-\tau \leq s \leq t$

Temos que $i \mapsto T(s, i) \tilde{Q}(i) \dot{\varphi}$ e $i \mapsto T(i, s) \tilde{F}(s) \dot{\varphi}$ são conininuos para cada $\phi \in C, \operatorname{logo} T(s, t) \tilde{Q}(t)$ e $T(t, s) \tilde{P}(s)$ são limitados em norma para todo $t \geq s$ em $\left[-\tau, t_{0}\right]$. Portanto, existe uma constante $N$ tal que:

$$
\begin{aligned}
& |T(s, t) \tilde{Q}(t)| \leq N e^{-\alpha(t-s)} \\
& |T(t, s) \tilde{P}(s)| \leq N e^{-\alpha(t-s)}
\end{aligned}
$$

Caso 3: $t_{0} \geq t \geq-\tau \geq s$

Temos:

$$
T(t, s) \tilde{P}(s)=T(t,-\tau) T(-\tau, s) \tilde{P}(s) \tilde{P}(s)=T(t,-\tau) \tilde{P}(-\tau) T(-\tau, s) \tilde{P}(s)
$$

logo,

$$
|T(t, s) \tilde{P}(s)| \leq N k k_{1} e^{-\alpha(t-s)}
$$

De modo análogo obtemos:

$$
|T(s, t) \tilde{Q}(t)| \leq N k k_{1} e^{-\alpha(t-s)}
$$

Portanto, tomando $K=\operatorname{máx}\left\{k k_{1}, N, N k k_{1}\right\}$ obtemos:

$$
\begin{aligned}
& |T(t, s) \tilde{P}(s)| \leq K e^{-\alpha(t-s)} \\
& |T(s, t) \tilde{Q}(t)| \leq K e^{-\alpha(t-s)}
\end{aligned}
$$

para todo $t \geq s$ em $\left(-\infty, t_{0}\right]$. 
Observação 2.3 Se tivermos atomicidade teremos a injetividade de $T(t, s)$, portanto a condição $T\left(t_{0},-\tau\right) \phi \neq 0$, para todo $0 \neq \phi \in C$.

Lema 2.7 Seja $J=[\tau,+\infty)$ e suponhamos que (1.2) possui uma dicotomia exponencial em J. Se $t_{0}<\tau$,

$$
X_{1}\left(t_{0}\right) \stackrel{\text { def }}{=}\left\{\phi: T\left(\tau, t_{0}\right) \phi \in \mathcal{R} P(\tau)\right\}
$$

e $X_{1}\left(t_{0}\right)$ possui a mesma codimensão de $\mathcal{R} P(\tau)$ então (1.2) possui uma dicotomia exponencial em $\left[t_{0},+\infty\right)$ com as projeções $\tilde{P}(t), \tilde{Q}(t) \rightarrow P(t), Q(t)$, exponencialmente quando $t \rightarrow+\infty$ na norma de operadores.

\section{Demonstração Seja}

$$
X_{1}(t)= \begin{cases}\mathcal{R} P(t) & t \geq \tau \\ \{\phi: T(\tau, t) \phi \in \mathcal{R} P(\tau)\} & t_{0} \leq t \leq \tau\end{cases}
$$

e $m=$ codimensão $X_{1}(\tau)=$ codimensão $X_{1}\left(t_{0}\right)$. Observemos que $X_{1}(t)$, para $t_{0} \leq t \leq \tau$, é uma subespaço fechado devido a continuidade de $T(\tau, t)$ e ao fato que $\mathcal{R P}(\tau)$ é um subespaço fechado de $C$. Tomemos um subespaço $m$-dimensional $X_{2}\left(t_{0}\right)$ tal que

$$
C=X_{1}\left(t_{0}\right) \oplus X_{2}\left(t_{0}\right)
$$

e

$$
X_{2}(t)=T\left(t, t_{0}\right) X_{2}\left(t_{0}\right), t \geq t_{0}
$$

Considere $\phi_{t_{0}} \in X_{2}\left(t_{0}\right)$. Se $\phi_{t_{0}} \neq 0, t \geq t_{0}$ então $T\left(t, t_{0}\right) \phi_{t_{0}} \neq 0$, pois caso contrário, isto é, $T\left(t, t_{0}\right) \phi_{t_{0}}=0 \in X_{1}(t)$ implicaria $\phi_{t_{0}} \in X_{1}\left(t_{0}\right)$. Logo $\phi_{t_{0}}=0$. Contradição! Portanto, $T\left(t, t_{0}\right) \phi_{t_{0}} \neq 0$ e $T\left(t, t_{0}\right)$ é um isomorfismo para todo $t \geq t_{0}$.

Temos também que:

$$
X_{2}(t)=T\left(t, t_{0}\right) X_{2}\left(t_{0}\right)=T(t, s)\left[T\left(s, t_{0}\right) X_{2}\left(t_{0}\right)\right]=T(t, s) X_{2}(s) .
$$

Logo, se $\phi_{s} \in X_{2}(s)$ satisfaz $T(t, s) \phi_{s}=0$, existe $\phi_{t_{0}} \in X_{2}\left(t_{0}\right)$ tal que $T\left(s, t_{0}\right) \phi_{t_{0}}=\phi_{s}$. Mas $T\left(t, t_{0}\right) \phi_{t_{0}}=0$, o que implica $\phi_{t_{0}}=0$. Portanto, $\phi_{s}=0 \mathrm{e}$ $T(t, s): X_{2}(s) \rightarrow X_{2}(t)$ é um isomorfismo. 
Mostremos agora que:

$$
C=X_{1}(t) \oplus X_{2}(t), \quad t \geq t_{0}
$$

(i) Seja $\phi_{t} \in X_{1}(t) \cap X_{2}(t)$. Como $\phi_{t} \in X_{2}(t)$ existe $\phi_{t_{0}} \in X_{2}\left(t_{0}\right)$ tal que $T\left(t, t_{0}\right) \phi_{t_{0}}=\phi_{t}$. Também $\phi_{t} \in X_{1}(t)$. Mas assim temos dois casos a considerar: quando $t_{0} \leq t \leq \tau$ e quando $t>\tau$.

- Se $t_{0} \leq t \leq \tau$, pela definição de $X_{1}(t)$ segue que $T(\tau, t) \phi_{t} \in \mathcal{R} P(\tau)$. Como $T(\tau, t)\left[T\left(t, t_{0}\right) \phi_{t_{0}}\right] \in X_{1}(\tau)$ obtemos $T\left(\tau, t_{0}\right) \phi_{t_{0}} \in X_{1}(\tau)$ logo $\phi_{t_{0}} \in X_{1}\left(t_{0}\right)$. Portanto, $\phi_{t_{0}}=0$ e $\phi_{t}=0$.

- Se $t>\tau, T(t, \tau)\left[T\left(\tau, t_{0}\right) \phi_{t_{0}}\right]=T\left(t, t_{0}\right) \phi_{t_{0}}=\phi_{t} \in X_{1}(t)=\mathcal{R} P(t)$. Então, $T\left(\tau, t_{0}\right) \phi_{t_{0}} \in \mathcal{R} P(\tau)$, o que implica que $\phi_{t_{0}} \in X_{1}\left(t_{0}\right)$ (lema 2.1). Portanto, $\phi_{t_{0}}=\phi_{t}=\mathbf{0}$.

(ii) $C=X_{1}(t)+X_{2}(t), t \geq t_{0}$

- Para $t \geq \tau$ sabemos codimensão $X_{1}(t)=\mathcal{R} P(t)$ é $m$, então;

$$
C .=X_{1}(t) \oplus X_{2}(t) \text {. }
$$

- Para $t_{0} \leq t \leq \tau$, seja $\phi \in C$ tal que $T(\tau, t) \phi=\phi_{1}+\phi_{2}$, com $\phi_{1} \in X_{1}(\tau)$ e $\phi_{2} \in X_{2}(\tau)$. Logo existe $\tilde{\phi}_{2} \in X_{2}(t)$ tal que $\dot{T}(\tau, t) \tilde{\phi}_{2}=\phi_{2}$ e $T(\tau, t)\left(\phi-\tilde{\phi}_{2}\right)=$ $\phi_{1} \in X_{1}(\tau) \operatorname{assim} \phi-\tilde{\phi}_{2} \in X_{1}(t)$ e $C=X_{1}(t)+X_{2}(t)$.

A decomposição $C=X_{1}(t) \oplus X_{2}(t)$ de $C$ define os operadores projeção $\tilde{P}(t)$ e $\tilde{Q}(t)=I-\tilde{P}(t)$ de modo que:

$$
\begin{aligned}
& \mathcal{R} \tilde{P}(t)=X_{1}(t) \\
& \mathcal{R} \tilde{Q}(t)=X_{2}(t)
\end{aligned}
$$

para $t \geq t_{0}$. Obviamente $X_{2}(s)$ é invariante sob $T(t, s)$ e $X_{1}(s)$ é invariante sob $T(t, s)$ pois:

- Se $t_{0} \leq s \leq t \leq \tau, T(\tau, t)\left[T(t, s) X_{1}(s)\right]=T(\tau, s) X_{1}(s) \subset \mathcal{R} P(\tau)$ o que implica $T(t, s) X_{1}(s) \subset X_{1}(t)$

- Se $t \geq s \geq \tau$ da hipótese de dicotomia segue a invariância de $X_{1}(s)$ sob $T(t, s)$; 
- E se $t_{0} \leq s \leq \tau \leq t, T(t, s) X_{1}(s)=T(t, \tau)\left[T(\tau, s) X_{1}(s)\right] \subset T(t, \tau) \mathcal{R} P(\tau)$ $\subset \mathcal{R} P(t)=X_{1}(t)$.

Segue então da invariância de $X_{1}(t)$ e $X_{2}(t)$ sob $T(t, s)$ que:

$$
\begin{aligned}
& T(t, s) \tilde{P}(s)=\tilde{P}(t) T(t, s) \\
& T(t, s) \tilde{Q}(s)=\tilde{Q}(t) T(t, s)
\end{aligned}
$$

para todo $t \geq s$ em $\left[t_{0},+\infty\right)$.

Neste ponto dividiremos o nosso estudo em três casos.

Caso 1: $t \geq \tau$

Como $\mathcal{R} P(t)=\mathcal{R} \tilde{P}(t), P(t) \tilde{P}(t)=\tilde{P}(t)$ e $\tilde{P}(t) P(t)=P(t)$,

$$
\begin{aligned}
\tilde{P}(t) & =\tilde{P}(t)(P(t)+Q(t)) \\
& =P(t)+\tilde{P}(t) Q(t) \\
& =P(t)+\tilde{P}(t) T(t, \tau) T(\tau, t) Q(t) \\
& =P(t)+T(t, \tau) \tilde{P}(\tau) T(\tau, t) Q(t) \\
& =P(t)+T(t, \tau) P(\tau) \tilde{P}(\tau) T(\tau, t) Q(t) .
\end{aligned}
$$

Portanto, $\tilde{P}(t)$ é fortemente contínuo para $t \geq \tau$ donde pelo Princípio da Limitação Uniforme obtemos $\tilde{P}(t)$ é limitado em norma para $t$ num subconjunto compacto de $[\tau,+\infty)$.

Das hipóteses (iii) e (iv) da definição de dicotomia obtemos:

$$
\begin{aligned}
|\tilde{P}(t)-P(t)| & \leq k e^{-\alpha(t-\tau)} M k e^{-\alpha(t-\tau)} \\
& \leq k^{2} M e^{-2 \alpha(t-\tau)}
\end{aligned}
$$

logo,

$$
|\tilde{P}(t)| \leq|\tilde{P}(t)-P(t)|+|P(t)| \leq k^{2} M e^{-2 \alpha(t-\tau)}+|P(t)|
$$

e, portanto, $|\tilde{P}(t)| \rightarrow|P(t)|$ exponencialmente em norma quando $t \rightarrow+\infty$.

Fazendo $s=t$ em $|T(t, s) P(s)| \leq k e^{-\alpha(t-s)}$ obtemos $|P(t)| \leq k$. Assim, $\tilde{Q}(t)$ também é fortemente contínuo e $|\tilde{Q}(t)| \rightarrow|Q(t)|$ exponencialmente em norma quando $t \rightarrow+\infty$. Além disso, $|Q(t)| \leq k$. Dai, $Q(t)$ e $\tilde{P}(t)$ são uniformemente limitadas em norma para $t \in[\tau,+\infty)$. Digamos: $|\tilde{P}(t)|,|\tilde{Q}(t)| \leq k_{1}$ para $t \geq \tau$. Então para $t \geq s \geq \tau$ :

$$
|T(t, s) \tilde{P}(s)|=|T(\dot{t}, s) P(s) \tilde{P}(s)| \leq k k_{1} e^{-\alpha(t-s)} .
$$


Mas,

$$
\begin{aligned}
T(t, s)[\tilde{Q}(s) T(s, t) Q(t) \tilde{Q}(t) \phi] & =\tilde{Q}(t) T(t, s) T(s, t) Q(t) \tilde{Q}(t) \phi \\
& =\tilde{Q}(t) Q(t) \tilde{Q}(t) \phi=\tilde{Q}(t) \phi \\
& =T(t, s)[T(s, t) \tilde{Q}(t) \phi]
\end{aligned}
$$

pois, $\tilde{Q}(t)=\tilde{Q}(t) Q(t)+\tilde{Q}(t) P(t)=\tilde{Q}(t) Q(t)$. Como $T(t, s)$ é um isomorfismo de $\mathcal{R} \tilde{Q}(s)$ sobre $\mathcal{R} \tilde{Q}(t)$ logo admite inversa que aplicada na igualdade acima nos dá:

$$
T(s, t) \tilde{Q}(t) \phi=\tilde{Q}(s) T(s, t) Q(t) \tilde{Q}(t) \phi
$$

portanto,

$$
|T(s, t) \tilde{Q}(t)| \leq k_{1}^{2} k e^{-\alpha(t-s)}, \quad t \geq s \geq \tau
$$

Caso 2: $t_{0} \leq t \leq \tau$

Como

$$
T(t, \tau) \tilde{Q}(\tau) T(\tau, t)=\tilde{Q}(t) T(t, \tau) T(\tau, t)=\tilde{Q}(t)
$$

obtemos a continuidade de $t \rightarrow \tilde{Q}(t) \phi$, para todo $\phi \in C$. Do Princípio da Limitaçäo Unitorme segue que $\tilde{Q}(t)$ é.uniformemente iimitadio em norma para $t \in\left[t_{0}, \tau\right]$. Logo, $T(s, t) \tilde{Q}(t)$ é limitado em norma para todo $t \geq s$ em $\left[t_{0}, \tau\right]$, portanto existe uma constante $N$ tal que:

$$
|T(s, t) \tilde{Q}(t)| \leq N e^{-\alpha(t-s)}
$$

Visto que $\tilde{P}(t)=I-\tilde{Q}(t)$ concluímos também que

$$
|T(t, s) \tilde{P}(s)| \leq N e^{-\alpha(t-s)}
$$

Caso 3: $t \geq \tau \geq s \geq t_{0}$

Temos:

$$
T(t, s) \tilde{P}(s)=T(t, \tau) T(\tau, s) \tilde{P}(s) \tilde{P}(s)=T(t, \tau) \tilde{P}(\tau) T(\tau, s) \tilde{P}(s)
$$

assim,

$$
|T(t, s) \tilde{P}(s)| \leq k k_{1} e^{-\alpha(t-\tau)} N e^{-\alpha(r-s)}=N k k_{1} e^{-\alpha(t-s)}
$$

De modo análogo obtemos:

$$
|T(s, t) \tilde{Q}(t)| \leq N k k_{1}^{2} e^{-\alpha(t-s)} .
$$


Portanto, tomando $K=\operatorname{máx}\left\{k k_{1}^{2}, k k_{1}, N, N k k_{1}, N k k_{1}^{2}\right\}$ segue

$$
\begin{aligned}
& |T(t, s) \tilde{P}(s)| \leq K e^{-\alpha(t-s)} \\
& |T(s, t) \tilde{Q}(t)| \leq K e^{-\alpha(t-s)}
\end{aligned}
$$

para todo $t \geq s$ em $\left[t_{0},+\infty\right)$.

As hipóteses no lema 2.7 são necessárias. Entretanto, um cálculo direto de $X_{1}\left(t_{0}\right)$ naquele lema é dificilmente exequível. Uma alternativa é considerar o sistema adjunto.

Lema 2.8 Suponhamos que a equação (1.2) possui uma dicotomia exponencial em $J=[\tau,+\infty)$. Seja $t_{0}<\tau$. Suponhamos que $T^{*}\left(t_{0}, \tau\right) \psi \neq 0$ para cada $0 \neq \psi \in \mathcal{R} Q^{*}(\tau)$. Então $T^{*}(s, t)$ possui uma dicotomia exponencial em $\left[t_{0},+\infty\right)$ $e$ as hipóteses do lema 2.7 estão satisfeitas.

Demonstração Pelo lema $2.4 T^{*}(s, t)$ possui uma dicotomia exponencial em $J$ e pelo lema 2.3 temos que $\operatorname{dim} \mathcal{R} Q^{*}(t)=\operatorname{dim}(\mathcal{R} Q(t))^{*}$ que por sua vez é igual a $\operatorname{dim} \mathcal{R} Q(t)=m$. Analogamente a demonstração do lema 2.6, mostra-se que $T^{*}(s ; t)$ possui uma dicotomia exponenciai sobre $\left[t_{0},+\infty j\right.$, com projeçöes $\tilde{F}^{*}(i)$ e $\tilde{Q}^{*}(t)$ relativamente a $\left[t_{0},+\infty\right)$. Temos também que $\mathcal{R} Q^{*}(t)=\mathcal{R} Q^{*}(t), t \geq \tau$ e $\operatorname{dim} \mathcal{R} \tilde{Q}^{*}(t)=m$ para todo $t \in\left[t_{0},+\infty\right)$.

É imediato que $\mathcal{R} \tilde{Q}^{*}(\tau)=[\mathcal{R} P(\tau)]^{0}$.

Seja $X_{1}\left(t_{0}\right)$ como no lema 2.7 .

Provemos que ${ }^{0}\left[\mathcal{R} \tilde{Q}^{*}\left(t_{0}\right)\right]=X_{1}\left(t_{0}\right)$. Para cada $\phi \in X_{1}\left(t_{0}\right)$, seja $\psi \in \mathcal{R} \tilde{Q}^{*}(\tau)=$ $[\mathcal{R} P(\tau)]^{0}$. Então, $\left\langle\psi, T\left(\tau, t_{0}\right) \phi\right\rangle=0$ pois $T\left(\tau, t_{0}\right) \phi \in \mathcal{R} P(\tau)$. Assim, $\left\langle T^{*}\left(\tau, t_{0}\right) \psi, \phi>=0\right.$, para todo $\psi \in \mathcal{R} \tilde{Q}^{*}(\tau)$. Também $T^{*}\left(t_{0}, \tau\right): \mathcal{R} \tilde{Q}^{*}(\tau) \rightarrow$ $\mathcal{R} \tilde{Q}^{*}\left(t_{0}\right)$ é sobrejetora. Dai, $T^{*}\left(t_{0}, \tau\right) \psi \in \mathcal{R} \tilde{Q}^{*}\left(t_{0}\right)$ e $\phi \in^{0}\left[\mathcal{R} \tilde{Q}^{*}\left(t_{0}\right)\right]$.

Reciprocamente, se $\phi \in^{0}\left[\mathcal{R} \tilde{Q}\left(t_{0}\right)\right]$ basta inverter o procedimento acima para mostrar que $T\left(\tau, t_{0}\right) \phi \in^{0}\left[\mathcal{R} Q^{*}(\tau)\right]=\mathcal{R} P(\tau)$. Portanto, $\phi \in X_{1}\left(t_{0}\right)$.

Finalmente, obtemos que:

$$
\begin{aligned}
\operatorname{codim} X_{1}\left(t_{0}\right) & =\operatorname{codim}^{0}\left[\mathcal{R} \tilde{Q}^{*}\left(t_{0}\right)\right]=\operatorname{codim} \mathcal{R} \tilde{P}\left(t_{0}\right) \\
& =\operatorname{dim} \mathcal{R} \tilde{Q}\left(t_{0}\right)=m=\operatorname{codim} \mathcal{R} P(\tau) . \square
\end{aligned}
$$

Observação 2.4 : As hipóteses no lema 2.8 podem ser verificadas considerando o sistema adjunto formal para (1.2), como veremos adiante. 


\section{Capítulo 3}

\section{Alternativa de Fredhom para Soluções Limitadas de Equações Diferenciais Funcionais}

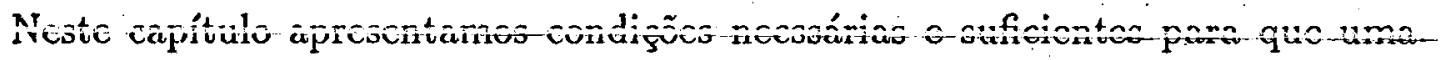
função $x: \mathbf{R}_{+}\left(\mathbf{R}_{-}\right) \rightarrow \mathbf{R}^{\mathbf{n}}$ seja solução de (1.1). Definimos um isomofismo entre as soluções (limitadas) do sitema adjunto verdadeiro e as soluções (limitadas) do sistema adjunto formal de (1.2) que utilizamos nas demonstrações do teorema 3.3 o qual fornece condições suficientes para a existência da dicotomia exponencial em $\mathbf{R}_{+}$e $\mathbf{R}_{-}$; e do teorema 3.4 que afirma que o operador

$$
\begin{aligned}
F: C_{b}^{\mathbf{1}}\left(\mathbf{R}, \mathbf{R}^{\mathbf{n}}\right) & \rightarrow C_{b}^{0}\left(\mathbf{R}, \mathbf{R}^{\mathbf{n}}\right) \\
x & \mapsto F(x)(t)=h(t)
\end{aligned}
$$

definido por:

$$
h(t)=\frac{d}{d t} x(t)-L(t) x_{t}
$$

é um operador de Fredholm cujo índice $I(F)=\operatorname{dim} \mathcal{R} Q^{-}(0)-\operatorname{dim} \mathcal{R} Q^{+}(0)$. Além disso, dá uma caracterização para o núcleo e para a imagem deste operador.

Para realizarmos a divisão induzida pela dicotomia exponencial na Fórmula da Variação das Constantes, consideramos a função matricial salto:

$$
X_{0}(\theta)= \begin{cases}0 & -r \leq \theta<0 \\ I & \theta=0\end{cases}
$$


A função $X_{0}$ desempenha um papel importante visto que a solução do sistema não homogêneo

$$
\left\{\begin{aligned}
\dot{x}(t) & =L(t) x_{t}+h(t) \\
x_{\sigma} & =\phi
\end{aligned}\right.
$$

onde $h: \mathbf{R} \rightarrow \mathbf{R}^{\mathbf{n}}$ é contínua, pode ser escrita como:

$$
x_{t}=T(t, \sigma) \phi+\int_{\sigma}^{t} T(t, s) X_{0} h(s) d s, \quad t \geq s .
$$

Como $X_{0}$ não é contínua, devemos estender nosso espaço para incluir as funções salto ou então dar significado as seguintes expressões envolvendo $X_{0}$ :

(1) $T(t, s) X_{0}$

(2) $\int_{a}^{b} T(t, s) X_{0} h(s) d s$;

(3) $<\psi, T(t, s) X_{0}>=<T^{*}(s, t) \psi, X_{0}>$;

(4) $\left\langle\psi, \int_{a}^{b} T(t, s) X_{0} h(s) d s\right\rangle=\int_{a}^{b}\left\langle\psi, T(t, s) X_{0}\right\rangle h(s) d s$;

(5) $P(s) X_{0}, Q(s) X_{0}$;

(6) $T(t, s) P(s) X_{0}=P(t) T(t, s) X_{0}$;

(7) $<\psi, T(t, s) P(s) X_{0}>=<T^{*}(s, t) P^{*}(t) \psi, X_{0}>$;

$(8)<\psi, T(s, t) Q(t) X_{0}>=\left\langle T^{*}(t, s) Q^{*}(s) \psi, X_{0}>\right.$

onde $\psi \in C^{*} \mathrm{e} t \geq s$ em $J$. Isto também será tratado neste capítulo.

Vamos supor que $\dot{x}(t)=L(t) x_{t}$ tem dicotomia exponencial com operadores projeção $P(t)$ e $Q(t)$ em $J$.

Os ítens de (1) a (3) foram discutidos nos capítulos 1 e (4) é uma aplicação do teorema de Fubini, isto é:

$$
\begin{aligned}
<\psi, \int_{a}^{b} T(t, s) X_{0} h(s) d s> & =\int_{-r}^{0}[d \psi(\theta)] \int_{a}^{b} T(t, s) X_{0}(\theta) h(s) d s \\
& =\int_{a}^{b}\left\{\int_{-r}^{0}[d \psi(\theta)] T(t, s) X_{0}(\theta)\right\} h(s) d s \\
& =\int_{a}^{b}<\psi, T(t, s) X_{0}>h(s) d s .
\end{aligned}
$$


Definição 3.1 Uma base invariante $\left\{q_{i}(t)\right\}\left(\left\{q_{i}^{*}(t)\right\}\right)$ em $\mathcal{R} Q(t)\left(\mathcal{R} Q^{*}(t)\right)$; $i=1,2, \ldots, m$; é uma base de $\mathcal{R} Q(t)\left(\mathcal{R} Q^{*}(t)\right)$ para cada $t \in J$, tal que

$$
q_{i}(t)=T(t, s) q_{i}(s) \quad\left(q_{i}^{*}(s)=T^{*}(s, t) q_{i}^{*}(t)\right)
$$

para todo $s \leq t$ em $J$.

Lema 3.1 Se $\Phi(t)=\left(q_{1}(t), q_{2}(t), \ldots, q_{m}(t)\right)$ e $\Psi(t)=\left(q_{1}^{*}(t), q_{2}^{*}(t), \ldots, q_{m}^{*}(t)\right)^{t}$ são bases invariantes de $\mathcal{R} Q(t)$ e $\mathcal{R} Q^{*}(t)$, respectivamente, entấo:

$$
\begin{aligned}
<\Psi(t), \Phi(t)> & =I_{m \times m} \\
Q(t) \phi & =\Phi(t)<\Psi(t), \phi> \\
P(t) \phi & =\phi-Q(t) \phi \\
Q^{*}(t) \psi & =<\psi, \Phi(i)>\Psi(t) \\
P^{*}(t) \psi & =\psi-Q^{*}(t) \psi
\end{aligned}
$$

para todo $t \in J, \phi \in C$ e $\psi \in C^{*}$.

Demonstração Seja $\left\{q_{j}\left(t_{0}\right)\right\}$ base de $\mathcal{R} Q\left(t_{0}\right), t_{0} \in J$, e consideremos $\left\{q_{i}^{*}\left(t_{0}\right)\right\}$ a

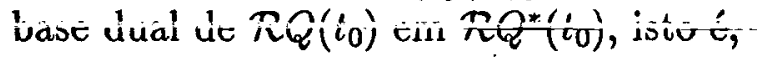

$$
<q_{i}^{*}\left(t_{0}\right), q_{j}\left(t_{0}\right)>=\delta_{i j} .
$$

Então

$$
\begin{aligned}
<q_{i}^{*}(t), q_{j}(t)> & =<q_{i}^{*}(t), T\left(t, t_{0}\right) q_{j}\left(t_{0}\right)> \\
& =<T^{*}\left(t_{0}, t\right) q_{i}^{*}(t), q_{j}\left(t_{0}\right)> \\
& =<q_{i}^{*}\left(t_{0}\right), q_{j}\left(t_{0}\right)> \\
& =\delta_{i j}
\end{aligned}
$$

para todo $t \geq t_{0}$ em $J$. Logo $\langle\Psi(t), \Phi(t)\rangle=I_{m \times m}$, para todo $t \in J$.

Como $Q(t) \phi \in \mathcal{R} Q(t), Q(t) \phi=\Phi(t) \alpha$ para todo $\phi \in C$ e $\alpha=\left(\alpha_{1}, \alpha_{2}, \ldots, \alpha_{m}\right)^{t} \in \mathbf{R}^{m}$ :

$$
\phi=Q(t) \phi+P(t) \phi=\Phi(t) \alpha+P(t) \phi
$$

e

$$
\begin{aligned}
\langle\Psi(t), \phi> & =\langle\Psi(t), \Phi(t) \alpha+P(t) \phi\rangle \\
& =<\Psi(t), \Phi(t)>\alpha+<\Psi(t), P(t) \phi> \\
& =\alpha+<P^{*}(t) \Psi(t), \dot{\phi}> \\
& =\alpha .
\end{aligned}
$$


Portanto,

$$
\begin{aligned}
& Q(t) \phi=\Phi(t)<\Psi(t), \phi> \\
& P(t) \phi=\phi-Q(t) \phi
\end{aligned}
$$

para todo $\phi \in C$.

De modo análogo prova-se que:

$$
\begin{aligned}
& Q^{*}(t) \psi=<\psi, \Phi(t)>\Psi(t) \\
& P^{*}(t) \psi=\psi+Q^{*}(t) \psi \cdot \square
\end{aligned}
$$

Temos então a definição:

Definição 3.2 Para todo $t \dot{\epsilon} J$, definimos:

$$
\begin{aligned}
Q(t) X_{0} & \left.=\Phi(t)<\Psi(t), X_{0}\right\rangle \\
P(t) X_{0} & =X_{0}-Q(t) X_{0} \\
Q(t) T(t, s) X_{0} & =\Phi(t)<\Psi(t), T(t, s) X_{0}> \\
P(t) \bar{T}(t, s) \bar{X}_{0} & =T(t, s) \bar{X}_{0}-\bar{Q}(t) T(t, s) \bar{X}_{0}
\end{aligned}
$$

Observe que $P(t) X_{0}$ não contínua.

Lema 3.2 Para todo $t \geq s$ em $J$,temos:

(i) $T(t, s) P(s) X_{0}=P(t) T(t, s) X_{0}$;

(ii) $<\psi, T(t, s) P(s) X_{0}>=<T^{*}(s, t) P^{*}(t) \psi, X_{0}>$;

(iii) $\left\langle\psi, T(s, t) Q(t) X_{0}>=<T^{*}(t, s) Q^{*}(s) \psi, X_{0}>\right.$.

\section{Demonstração}

(i) Como $\Phi(t)$ e $\Psi(t)$ são bases invariantes de $\mathcal{R} Q(t)$ e $\mathcal{R} Q^{*}(t)$, respectivamente, temos:

$$
\begin{aligned}
T(t, s) Q(s) X_{0} & =T(t, s) \Phi(s)<\Psi(s), X_{0}> \\
& =\Phi(t)<T^{*}(s, t) \Psi(t), X_{0}> \\
& =\Phi(t)<\Psi(t), T(t, s) X_{0}> \\
& =Q(t) T(t, s) X_{0} .
\end{aligned}
$$


(ii) Para todo $\psi \in C^{*}$ e $t \geq s$ em $J$ segue

$$
\begin{aligned}
<\psi, Q(t) X_{0}> & =<\psi, \Phi(t)<\Psi(t), X_{0}>> \\
= & <\psi, \Phi(t)><\Psi(t), X_{0}> \\
= & <\left\langle\psi, \Phi(t)>\Psi(t), X_{0}>\right. \\
= & <Q^{*}(t) \psi, X_{0}>.
\end{aligned}
$$

De $Q(s) X_{0} \in C$ e pela igualdade acima

$$
\begin{aligned}
\left\langle\psi, T(t, s) Q(s) X_{0}>\right. & =<T^{*}(s, t) \psi, Q(s) X_{0}> \\
& =<Q^{*}(s) T^{*}(s, t) \psi, X_{0}>
\end{aligned}
$$

tem-se

$$
\begin{aligned}
<\psi, T(t, s) P(s) X_{0}> & =<\psi, T(t, s) X_{0}-T(t, s) Q(s) X_{0}> \\
& =<\psi, T(t, s) X_{0}>-<\psi, T(t, s) Q(s) X_{0}> \\
& =<T^{*}(s, t) \psi, X_{0}>-<Q^{*}(s) T^{*}(s, t) \psi, X_{0}> \\
= & <T^{*}(s, t) \psi-Q^{*}(s) T^{*}(s, t) \psi, X_{0}> \\
= & <P^{*}(s) T^{*}(s, t) \psi, X_{0}>
\end{aligned}
$$

e como $T^{*}(s, t)$ tem dicotomia exponencial sobre $J$

$$
<\psi, T(t, s) P(s) X_{0}>=<T^{*}(s, t) P^{*}(t) \psi, X_{0}>.
$$

(iii) Usando que $\left.T(t, s)\right|_{\mathcal{R} Q(s)}$ e $\left.T^{*}(s, t)\right|_{\mathcal{R Q} Q^{*}(t)}$ são isomorfismos obtemos:

$$
\begin{aligned}
<\psi, T(s, t) Q(t) X_{0}> & =\left\langle\psi, Q(s) T(s, t) Q(t) X_{0}>\right. \\
& =<Q^{*}(t) T^{*}(t, s) Q^{*}(s) \psi, X_{0}> \\
& =<T^{*}(t, s) Q^{*}(s) \psi, X_{0}>. \square
\end{aligned}
$$

Lema 3.3 Para todo $t \geq s$ em $J$, existem constantes $K$ e $\alpha$ tais que

$$
\begin{aligned}
\left|T(s, t) Q(t) X_{0}\right| & \leq K e^{-\alpha(t-s)} \\
\sup _{\theta \in[-r, 0]}\left|\left(T(t, s) P(s) X_{0}\right)(\theta)\right| & \leq K e^{-\alpha(t-s)}
\end{aligned}
$$

Demonstração Seja $\psi \in C^{*}=B_{0}[-r, 0]$ e $\|\psi\|_{B_{0}}=1$. Como $T^{*}(s, t)$ tem dicotomia sobre $J$,

$$
\begin{aligned}
\left|<\psi, T(t, s) P(s) X_{0}>\right| & =\left|<T^{*}(s, t) P^{*}(t) \psi, X_{0}>\right| \\
& \leq\left|T^{*}(s, t) P^{*}(t) \psi\right|_{B_{0}} \\
& \leq K e^{-\alpha(t-s)}
\end{aligned}
$$




$$
\begin{aligned}
\mid<\psi, T(s, t) Q(t) X_{0}>1 & =\mid<T^{*}(t, s) Q^{*}(s) \psi, X_{0}>1 \\
& \leq\left|T^{*}(t, s) Q^{*}(s) \psi\right|_{B_{0}} \\
& \leq K e^{-\alpha(t-s)} .
\end{aligned}
$$

Logo do teorema de Hahn-Banach segue que

$$
\begin{aligned}
\left|T(s, t) Q(t) X_{0}\right| & \leq K e^{-\alpha(t-s)} \\
\sup _{\theta \in[-r, 0]}\left|\left(T(t, s) P(s) X_{0}\right)(\theta)\right| & \leq K e^{-\alpha(t-s)}
\end{aligned}
$$

$t \geq s \mathrm{em} J$

Observação 3.1 Apesar de $T(t, s) P(s) X_{0}$ não ser um elemento de $C$ para $s \leq$ $t \leq s+r$, escreveremos a segunda igualdade do lema 3.3 como

$$
\cdot T(t, s) P(s) X_{0} \mid \leq K e^{-\alpha(t-s)} .
$$

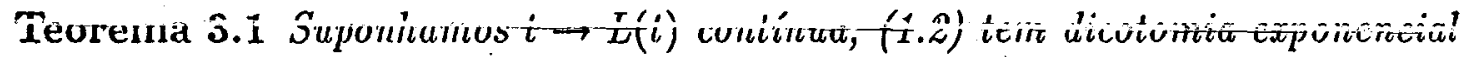
em $\mathbf{R}_{+}$. Então para qualquer função $h: \mathbf{R}_{+} \rightarrow \mathbf{R}^{\mathbf{n}}$ contínua e limitada sobre $\mathbf{R}_{+}$, $x: \mathbf{R}_{+} \rightarrow \mathbf{R}^{\mathbf{n}}$ é uma solução limitada de (1.1) se, e somente se

$x_{t}=T(t, 0) P(0) \phi+\int_{0}^{t} T(t, s) P(s) X_{0} h(s) d s-\int_{t}^{\infty} T(t, s) Q(s) X_{0} h(s) d s, \quad t \geq 0$.

Demonstração Se $x(t)$ é solução de (1.1), limitada em $\mathbf{R}_{+}$, pela Fórmula da Variação das Constantes

$$
x_{t}=T(t, \tau) x_{\tau}+\int_{\tau}^{t} T(t, s) X_{0} h(s) d s, \quad t \geq \tau \geq 0
$$

logo,

$$
\begin{array}{ll}
Q(t) x_{t}=T(t, \tau) Q(\tau) x_{\tau}+\int_{\tau}^{t} T(t, s) Q(s) X_{0} h(s) d s, & t \geq \tau \geq 0 \\
T(\tau, t) Q(t) x_{t}=Q(\tau) x_{\tau}+\int_{\tau}^{i} T(\tau, s) Q(s) X_{0} h(s) d s, & t \geq \tau \geq 0 .
\end{array}
$$

Fazendo $t \rightarrow+\infty$ na expressão acima obtemos:

$$
\left|T(\tau, t) Q(t) x_{t}\right| \leq K e^{-\alpha(t-\tau)}\left|x_{t}\right|
$$


Como $\left|x_{t}\right|$ é limitado temos

$$
Q(\tau) x_{\tau}=-\int_{\tau}^{\infty} T(\tau, s) Q(s) X_{0} h(s) d s
$$

Ainda,

$$
P(t) x_{t}=T(t, 0) P(0) \phi+\int_{0}^{t} T(t, s) P(s) X_{0} h(s) d s .
$$

Tomando em (3.1) $\tau=t$ e somando a (3.2) obtemos:

$x_{i}=T(t, 0) P(0) \phi+\int_{0}^{t} T(t, s) P(s) X_{0} h(s) d s-\int_{t}^{\infty} T(t, s) Q(s) X_{0} h(s) d s, \quad t \geq 0$.

Resta mostrar que se

$$
x_{t}=T(t, 0) P(0) \phi+\int_{0}^{t} T(t, s) P(s) X_{0} h(s) d s-\int_{t}^{\infty} T(t, s) Q(s) X_{0} h(s) d s, \quad t \geq 0
$$

então $x_{t}$ é solução de (1.1), limitada em $\mathbf{R}_{+}$. De fato, a limitação segue de:

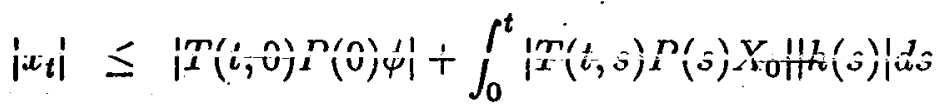

$$
\begin{aligned}
& +\int_{t}^{\infty}\left|T(t, s) Q(s) X_{0} \| h(s)\right| d s \\
& \leq K e^{-\alpha t}|\phi|+\frac{M K}{\alpha}\left(1-e^{-\alpha t}\right)+\frac{K M}{\alpha}
\end{aligned}
$$

logo,

$$
\left|x_{t}\right| \leq K|\phi|+\frac{2 M K}{\alpha}, \quad t \geq 0
$$

onde $M>0$ e $|h(s)| \leq M$.

Para ver que

$$
x_{t}=T(t, 0) P(0) \phi+\int_{0}^{t} T(t, s) P(s) X_{0} h(s) d s-\int_{t}^{\infty} T(t, s) Q(s) X_{0} h(s) d s, \quad t \geq 0
$$

é solução de (1.1) notemos que, para $t \geq \tau \geq 0$

$$
\begin{aligned}
T(t, \tau) x_{\tau}= & T(t, \tau) T(\tau, 0) P(0) \phi+T(t, \tau) \int_{0}^{\tau} T(\tau, s) P(s) X_{0} h(s) d s \\
& -T(t, \tau) \int_{\tau}^{\infty} T(\tau, s) Q(s) X_{0} h(s) d s \\
= & T(t, 0) P(0) \phi+\int_{0}^{t} T(t, s) P(s) X_{0} h(s) d s-\int_{t}^{\infty} T(t, s) Q(s) X_{0} h(s) d s
\end{aligned}
$$




$$
\begin{aligned}
& -\int_{\tau}^{t} T(t, s) P(s) X_{0} h(s) d s-\int_{\tau}^{t} T(t, s) Q(s) X_{0} h(s) d s \\
= & T(t, 0) P(0) \phi+\int_{0}^{t} T(t, s) P(s) X_{0} h(s) d s \\
& -\int_{t}^{\infty} T(t, s) Q(s) X_{0} h(s) d s-\int_{\tau}^{t} T(t, s) X_{0} h(s) d s \\
= & x_{t}-\int_{\tau}^{t} T(t, s) X_{0} h(s) d s
\end{aligned}
$$

ou seja,

$$
x_{t}=T(t, \tau) x_{\tau}+\int_{\tau}^{t} T(t, s) X_{0} h(s) d s, \quad t \geq \tau . \square
$$

Teorema 3.2 Suponhamos que $t \rightarrow L(t)$ continua, (1.2) tem dicotomia expo-

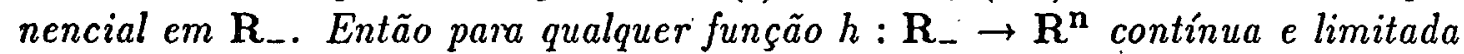
em $\mathbf{R}_{-}, x: \mathbf{R}_{-} \rightarrow \mathbf{R}^{\mathbf{n}}$ é solução limitada de (1.1) se, e somente se

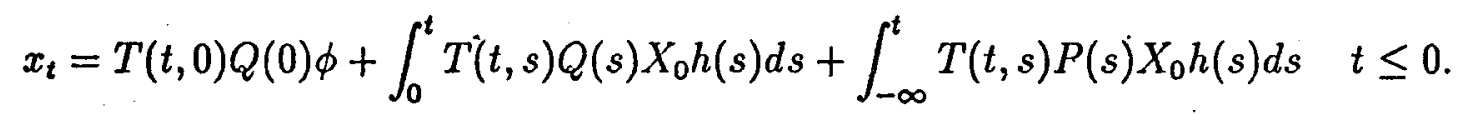

Demonstração Se $x: \mathbf{R}_{-} \rightarrow \mathbf{R}^{\mathbf{n}}$ é solução de (1.1) então pela Fórmula da Variação das Constantes

$$
x_{t}=T(t, \tau) x_{\tau}+\int_{\tau}^{t} T(t, s) X_{0} h(s) d s \quad 0 \geq t \geq \tau
$$

logo

$$
P(t) x_{t}=T(t, \tau) P(\tau) x_{\tau}+\int_{\tau}^{t} T(t, s) P(s) X_{0} h(s) d s
$$

fazendo $\tau \rightarrow-\infty$ obtemos

$$
P(t) x_{t}=\int_{-\infty}^{t} T(t, s) P(s) X_{0} h(s) d s .
$$

Ainda

$$
Q(0) \phi=T(0, t) Q(t) x_{t}+\int_{t}^{0} T(0, s) Q(s) X_{0} h(s) d s
$$

então

$$
\begin{gathered}
T(t, 0) Q(0) \phi=Q(t) x_{t}+\int_{t}^{0} T(t, s) Q(s) X_{0} h(s) d s \\
Q(t) x_{t}=T(t, 0) Q(0) \phi-\int_{t}^{0} T(t, s) Q(s) X_{0} h(s) d s
\end{gathered}
$$


Somando (3.3) com (3.4) obtemos:

$x_{t}=T(t, 0) Q(0) \phi+\int_{0}^{t} T(t, s) Q(s) X_{0} h(s) d s+\int_{-\infty}^{t} T(t, s) P(s) X_{0} h(s) d s, \quad t \leq 0$.

Resta mostar que

$x_{t}=T(t, 0) Q(0) \phi+\int_{0}^{t} T(t, s) Q .(s) X_{0} h(s) d s+\int_{-\infty}^{t} T(t, s) P(s) X_{0} h(s) d s, \quad t \leq 0$ é solução limitada de (1.1) em $\mathbf{R}_{\text {. }}$. Notemos que para $0 \geq t \geq \tau$

$$
\begin{aligned}
T(t, \tau) x_{\tau}= & T(t, \tau) T(\tau, 0) Q(0) \phi+T(t, \tau) \int_{0}^{\tau} T(\tau, s) Q(s) X_{0} h(s) d s \\
& +T(t, \tau) \int_{-\infty}^{\tau} T(\tau, s) P(s) X_{0} h(s) d s \\
= & T(t, 0) Q(0) \phi-\int_{\tau}^{0} T(t, s) Q(s) X_{0} h(s) d s+\int_{-\infty}^{\tau} T(t, s) P(s) X_{0} h(s) d s \\
= & T(t, 0) Q(0) \phi-\int_{t}^{0} T(t, s) Q(s) X_{0} h(s) d s+\int_{-\infty}^{t} T(t, s) P(s) X_{0} h(s) d s \\
& -\int_{\tau}^{t} T(t, s) Q(s) X_{0} h(s) d s-\int_{\tau}^{t} T(t, s) P(s) X_{0} h(s) d s \\
= & x_{t}-\int_{\tau}^{t} T(t, s) X_{0} h(s) d s .
\end{aligned}
$$

ou seja,

$$
x_{t}=T(t, \tau) x_{\tau}+\int_{\tau}^{t} T(t, s) X_{0} h(s) d s, \quad 0 \geq t \geq \tau
$$

Para ver que $x_{t}$ é limitada em $R_{-}$, notemos que:

$$
\begin{aligned}
\left|x_{t}\right| & \leq K e^{\alpha t}|\phi|+\int_{t}^{0} K M e^{-\alpha(s-t)} d s+\int_{-\infty}^{t} K e^{-\alpha(t-s)} M d s \quad t \leq 0 \\
& \leq K e^{\alpha t}|\phi|+\frac{K M}{\alpha}\left(1-e^{\alpha t}\right)+\frac{K M}{\alpha} \\
& \leq K|\phi|+\frac{2 K M}{\alpha}
\end{aligned}
$$

onde $M>0$ e $|h(s)|<M$, ou seja,

$$
\left|x_{i}\right| \leq K|\phi|+\frac{2 K M}{\alpha}, \quad t \leq 0 . \square
$$

Definição 3.3 Consideremos o operador

$$
\begin{aligned}
\tilde{\Psi}: B V\left([\sigma, t+r], \mathbf{R}^{\mathbf{n}^{*}}\right) & \rightarrow C\left([\sigma, t], B_{0}[-r, 0]\right) \\
\boldsymbol{y} & \mapsto \tilde{\Psi}(y)
\end{aligned}
$$

onde: 
- $B V\left([\sigma, t+r], \mathbf{R}^{\mathbf{n}^{*}}\right)$ é o espaço vetorial das funções de variação limitada em $[\sigma, t+r]$ com valores em $\mathbf{R}^{\mathbf{n}^{*}}$ e contínuas em $[\sigma, t+r]$.

- $C\left([\sigma, t], B_{0}[-r, 0]\right)$ é o conjunto das funções contínuas definidas em $[\sigma, t]$ com valores em $B_{0}[-r, 0]$.

$\tilde{\Psi}$ é definida como:

$$
\tilde{\Psi}(y)(u)=\psi(u, .)
$$

onde

$$
\begin{aligned}
\psi(u, 0) & =0 \\
\psi(u, s-u) & =\int_{u}^{u+r} y(\alpha) p(\alpha, s) d \alpha-\int_{u}^{u+r} y(\alpha) p(\alpha, u) d \alpha-y(u)
\end{aligned}
$$

Observação 3.2 Quando $h=0$, a fórmula dada pelo lema 1.5 pode ser escrita como:

$$
\left.<\psi(t, .), x_{t}\right\rangle=<\psi(s, .), x_{s}>
$$

se considerarmos $x$ solução de (1.1) e y solução de (1.6).

De fato,

$$
\begin{aligned}
\left\langle\psi(t, .), x_{t}\right\rangle= & \int_{-r}^{0} d_{\theta} \psi(t, 0) x(t+\theta) \\
= & \int_{t-r}^{t} d_{s} \psi(t, s-t) x(s) \\
= & \int_{t-r}^{t^{-}} d_{s} \psi(t, s-t) x(s)+\int_{t^{-}}^{t} d_{s} \psi(t, s-t) x(s) \\
= & \int_{t-r}^{t^{-}} d_{s}\left\{\int_{t}^{t+r} y(\alpha) p(\alpha, s) d \alpha-\int_{t}^{t+r} y(\alpha) p(\alpha, t) d \alpha-y(t)\right\} x(s) \\
& +\left[\psi(t, 0)-\psi\left(t, 0^{-}\right)\right] x(t) \\
= & \int_{t-r}^{t-} d_{s}\left\{\int_{t}^{t+r} y(\alpha) p(\alpha, s) d \alpha\right\} x(s)+y(t) x(t)
\end{aligned}
$$

Consequentemente,

$$
\psi(s, .)=T^{*}(s, t) \psi(t, .)
$$

isto é, $\psi(s,$.$) é solução do sistema adjunto verdadeiro.$ 
Lema 3.4 Seja $J=\left[t_{0},+\infty\right)$ ou $(-\infty,+\infty)$.

Sejam:

$$
\begin{aligned}
Y_{1}= & \{y: y(t) \text { satisfaz o sistema adjunto formal }(1.6) \text { em } J, \text { isto é, } \\
& \left.y_{s}=\tilde{T}(s, t) y_{t}, s \leq t \text { em } J\right\} \\
\Psi_{1}= & \{\psi(., \theta),-r \leq \theta \leq 0: \psi(s, .) \text { satisfaz o sistema adjunto verdadeiro } \\
& \text { em.J, isto é, } \left.\psi(s, .)=T^{*}(s, t) \psi(t, .), s \leq t \text { em } J\right\}
\end{aligned}
$$

Então $\left.\tilde{\Psi}\right|_{Y_{1}}: Y_{1} \rightarrow \Psi_{1}, u \in J$, definida em (3.5) é um isomorfismo linear cuja inversa $\left(\left.\Psi\right|_{\Psi_{1}}\right)^{-1}: \Psi_{1} \rightarrow Y_{1}, u \in J$, é dada por:

$$
\psi\left(\alpha, 0^{-}\right)=-y(\alpha), \quad \alpha \in[u, u+r]
$$

onde:

$$
\begin{aligned}
\psi(\alpha, .) & =T^{*}(\alpha, u+r) \psi(u+r, .) e \\
\psi\left(\alpha, 0^{-}\right) & =\lim _{\theta \rightarrow 0^{-}} \psi(\alpha, \theta)
\end{aligned}
$$

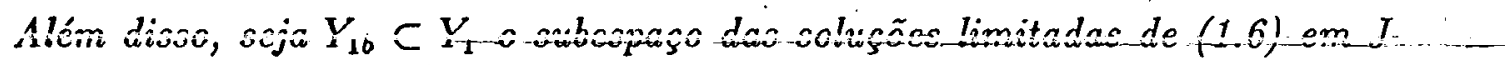
com a norma

$$
|y|_{Y_{1 b}}=\sup _{s \in J}|y(s)|_{\mathbf{R}^{\mathbf{n}^{*}}}
$$

e seja $\Psi_{1 b} \subset \Psi$ o subespaço das soluções limitadas do sistema adjunto verdadeiro em $J$ com a norma

$$
|\psi(., .)|_{\Psi_{1 b}}=\sup _{s \in J}|\psi(s, .)|_{B_{0}}
$$

então $\left.\tilde{\Psi}\right|_{Y_{1 b}} \quad \dot{e} \quad$ um isomorfismo topológico de $Y_{1 b}$ sobre $\Psi_{1 b}$ se $\int_{u}^{u+r}|L(\alpha)| d \alpha \leq M, u \in J$, para alguma constante $M>0$.

Demonstração Notemos que pela observação $(3.2)$ que $\psi(u,$.$) como definido em$ (3.5) satisfaz o sistema adjunto verdadeiro.

Provemos primeiramente que $\left.\tilde{\Psi}\right|_{Y_{1}}$ é injetora. Suponhamos $\left.\tilde{\Psi}\right|_{Y_{1}}(y)(u)=0$, para todo $u \in J$, ou seja, $\psi(u,)=$.0 para todo $u \in J$. Tomando o limite em $s$ quando $s \rightarrow u^{-}$obtemos:

$$
0=\lim _{s \rightarrow u^{-}} \psi(u, s-u)=-y(u)
$$

isto é, $y(u)=0$ para todo $u \in J$. 
Tomemos $\psi(\alpha,.) \in B_{0}, \alpha \in[u, u+r], u \in J$. Pela demonstração do teorema 1.7 temos que:

$$
\begin{aligned}
\langle\psi(\alpha, .), \phi\rangle & =\left\langle T^{*}(\alpha, u+r) \psi(u+r, .) ; \phi\right\rangle \\
& =\int_{-r}^{0^{-}} d_{\xi}[\psi(u+r, \xi+\alpha-(u+r))] \phi(\xi) \\
+\int_{-r}^{0^{-}} d_{\xi} & {\left[\int_{\alpha}^{u+r} y(\beta, u+r) p(\beta, \alpha+\xi) d \beta\right] \phi(\xi)+y(\alpha, u+r) \phi(0) }
\end{aligned}
$$

onde

$$
y(\alpha, u+r)= \begin{cases}0 & \alpha>u+r \\ \int_{\alpha-u-r}^{0}\left[d_{\theta} \psi(u+r, \theta)\right] Y(\alpha, u+r+\theta) & \alpha \leq u+r\end{cases}
$$

satisfaz

$$
y(\alpha, u+r)+\int_{\alpha}^{u+r} y(\beta, u+r) p(\beta, \alpha) d \beta=-\psi(u+r, \alpha-(u+r)), \quad \alpha \leq u+r
$$

e é a solução da equação àdjunta formal

$$
v(\alpha, u+r)+\int_{\alpha}^{u+r} y(\beta, u+r) \eta(\beta, \alpha) d \beta=-y(n+r,-r) \alpha \leq u
$$

Disso obtemos:

$$
\begin{aligned}
\dot{\psi\left(\alpha, 0^{-}\right)=} & -y(\alpha, u+r) \stackrel{\text { def }}{=}-y(\alpha) \\
\psi(\alpha, \theta)= & T^{*}(\alpha, u+r) \psi(u+r, \theta) \\
= & \int_{\alpha}^{u+r} y(\beta, u+r) p(\beta, \alpha+\theta) d \beta \\
& +\psi(u+r, \theta+\alpha-(u+r))
\end{aligned}
$$

para $-r \leq \theta<0$ e $\alpha \in[u, u+r]$.

Então, dado $\psi(\alpha,.) \in B_{0}$, existe $y \in Y_{1}, y(\alpha)=-\psi\left(\alpha, 0^{-}\right), \alpha \in[u, u+r]$ tal que

$$
\begin{aligned}
\tilde{\Psi}(y)(u)= & \int_{u}^{u+r}\left[-\psi\left(\alpha, 0^{-}\right)\right] p(\alpha, s) d \alpha-\int_{u}^{u+r}\left[-\psi\left(\alpha, 0^{-}\right)\right] p(\alpha, u) d \alpha \\
& -\left[-\psi\left(u, 0^{-}\right)\right] \\
= & \int_{u}^{u+r}\left[-\psi\left(\alpha, 0^{-}\right)\right] p(\alpha, s) d \alpha+\psi(u+r,-r) \\
= & \psi(u, s-u)
\end{aligned}
$$

E, portanto, $\left.\tilde{\Psi}\right|_{\gamma_{1}}$ é sobrejetora. 
Provemos que $\left.\tilde{\Psi}\right|_{Y_{1} b}$ é um isomorfismo topológico se $\int_{u}^{u+r}|L(\alpha)| d \alpha \leq M$.

É imediato que :

$$
\psi(., .) \mapsto\left(\left.\tilde{\Psi}\right|_{\Psi_{1}}\right)^{-1}(\psi(., .))=-\psi\left(., 0^{-}\right)
$$

é contínua.

Reciprocamente,

$$
\begin{aligned}
|\psi(u, .)|_{B_{0}} & =|y(u)|+V_{-r}^{0}\left(\int_{u}^{u+r} y(\alpha) p(\alpha, u+\theta) d \alpha\right) \\
& \leq|y(u)|+\int_{u}^{u+r}|y(\alpha)||p(\alpha, u+\theta)|_{B_{0}} d \alpha \\
& \leq|y|_{Y_{2 b}}+|y|_{Y_{1 b}} \int_{u}^{u+r}|L(\alpha)| d \alpha \\
& \leq(M+1)|y|_{Y_{1 b}} . \square
\end{aligned}
$$

Observação 3.3 Lembremos que a solução nula é um ponto de equilibrio hiperbólicn de (1.2) se sun equncoñn característica não possui raizes sobre o eixo imaginário o que implica que a equação acima tem dicotomia exponencial em $\mathbf{R}$.

Teorema 3.3 Seja $L(t)$ como em (1.2) e $L(t) \rightarrow L^{ \pm}$quando $t \rightarrow \pm \infty$. Suponhamos $\dot{x}(t)=L^{ \pm} x_{i}$ possui zero como um ponto de equilibrio hiperbólico com $P^{ \pm}$ projeções sobre o subespaço estável. Suponhamos $T(t, s) \phi \neq 0$ para $s \leq t$ em $\mathbf{R}_{-}$, $0 \neq \phi \in C, e \tilde{T}(s, t) \psi \neq 0$ para $s \leq t$ em $[-r, \infty), 0 \neq \psi \in B_{0}$. Então (1.2) possui uma dicotomia exponencial em $\mathbf{R}_{-} e \mathbf{R}_{+}$com as projeçôes $P^{ \pm}(t) \rightarrow P^{ \pm}$ quando $t \rightarrow \pm \infty$.

Demonstração Como $\dot{x}(t)=L^{ \pm} x_{t}$ possui zero como ponto de equilíbrio hiperbólico e $L(t) \rightarrow L^{ \pm}$quando $t \rightarrow \pm \infty$, pelo lema 2.5, (1.2) tem dicotomia exponencial em $[\tau,+\infty),(-\infty,-\tau]$ para alguma constante $\tau>0$ com projeções $P^{+}(t)$ e $P^{-}(t)$, respectivamente, com $P^{+}(t), P^{-}(t) \rightarrow P^{+}, P^{-}$. Logo (1.2) tem dicotomia exponencial em $(-\infty, 0]$ com projeções $P(t) \rightarrow P^{-}(t)$ quando $t \rightarrow-\infty$ pois para $0 \neq \phi \in \mathcal{R} Q(-\tau), T(0,-\tau) \phi \neq 0$ logo pelo lema 2.6 (1.2) tem dicotomia exponencial em $(-\infty, 0]$.

Além disso, para todo $0 \neq \eta_{r} \in \mathcal{R} Q^{*}(\tau)$

$$
T^{*}(0, \tau) \eta_{\tau}=\tilde{\Psi}_{0}(y) \neq 0
$$


desde que $\tilde{T}(s, t) \psi \neq 0$ para $s \leq t$ em $[-r, \infty), \psi \neq 0$ em $B_{0}$. Pelo lema 2.8 $T^{*}(s, t)$ tem dicotomia exponencial em $[0,+\infty)$ e daí as hipóteses do lema 2.7 estão satisfeitas de modo que quando consideramos o conjunto

$$
X_{1}(0)=\{\phi: T(\tau, 0) \phi \in \mathcal{R} P(\tau)\}
$$

com $\tau>0$ concluímos que (1.2) tem dicotomia exponencial em $[0,+\infty)$ com projeções $P(t) \rightarrow P^{+}(t)$ quando $t \rightarrow+\infty$.

Seja $C_{b}^{0}\left(\mathbf{R}, \mathbf{R}^{\mathbf{n}}\right)$ o espaço das funções contínuas e limitadas com a norma do sup e seja $C_{b}^{\mathbf{1}}\left(\mathbf{R}, \mathbf{R}^{\mathbf{n}}\right)$ o espaço. das funções em $C_{b}^{0}\left(\mathbf{R}, \mathbf{R}^{\mathbf{n}}\right)$ com derivadas em $C_{b}^{0}\left(\mathbf{R}, \mathbf{R}^{\mathbf{n}}\right)$ cuja norma é dada por $|x|_{C_{b}^{1}}=|x|_{C_{b}^{0}}+|\dot{x}|_{C_{b}^{0}}, x \in C_{b}^{1}\left(\mathbf{R}, \mathbf{R}^{\mathbf{n}}\right)$. Definições semelhantes são dadas substituindo $\mathbf{R}^{\mathbf{n}}$ por $\mathbf{R}^{\mathbf{n}^{*}}$ :

Definição 3.4 Consideremos o operador:

$$
\begin{aligned}
F: C_{b}^{\mathbf{1}}\left(\mathbf{R}, \mathbf{R}^{\mathbf{n}}\right) & \rightarrow C_{b}^{0}\left(\mathbf{R}, \mathbf{R}^{\mathbf{n}}\right) \\
x & \mapsto F(x)(t)=h(t)
\end{aligned}
$$

äefiniảo por:

$$
h(t)=\frac{d}{d t} x(t)-L(t) x_{t}
$$

Teorema 3.4 Seja $L(t)$ contínua na norma do operador com respeito a $t \in \mathbf{R} e$ $|L(u)|<M$ para todo $u \in \mathbf{R}$. Suponhamos que (1.2) possui dicotomia exponencial em $\mathbf{R}_{+}$e $\mathbf{R}_{-}$com' projeçóes $P^{+}(t)$ e $P^{-}(t)$, respectivamente. Então o operador acima é um operador de Fredholm de índice $I(F)=\operatorname{dim} \mathcal{R} Q^{-}(0)-\operatorname{dim} \mathcal{R} Q^{+}(0)$. Além disso,

$$
\begin{aligned}
\mathcal{N}(F)= & \left\{(T(t, 0) \phi)(0): \phi \in \mathcal{R} P^{+}(0) \cap \mathcal{R} Q^{-}(0), t \in \mathbf{R}\right\} \\
\mathcal{R}(F)= & \left\{h: h \in C_{b}^{0}\left(\mathbf{R}, \mathbf{R}^{\mathbf{n}}\right), \int_{-\infty}^{+\infty} y(t) h(t) d t=0\right. \text { para todo } \\
& \left.y \in C_{b}^{0}\left(\mathbf{R}, \mathbf{R}^{\mathbf{n}}\right) \text { satisfazendo o sistema adjunto formal }(1.6)\right\}
\end{aligned}
$$

Demonstração Do teorema 2.1 temos que:

$$
\begin{aligned}
& \mathcal{R} P^{+}(0)=\{\phi \in C: T(t, 0) \phi \text { é limitada para } t \geq 0\} \\
& \mathcal{R} Q^{-}(0)=\{\phi \in C: T(t, 0) \phi \text { está definida é é limitada para } t \leq 0\}
\end{aligned}
$$


logo, se $T(t, 0) \phi$ é limitada para todo $t \in \mathbf{R}, \phi \in \mathcal{R} P^{+}(0) \cap \mathcal{R} Q^{-}(0)$.

$x \in C_{b}^{0}\left(\mathbf{R}, \mathbf{R}^{\mathbf{n}}\right)$ é um elemento de $\mathcal{N}(F)$ se, e só se, $x$ é solução de (1.2),isto é, $\mathcal{N}(F)$ é o conjunto de todas as soluções limitadas de (1.2) que são escritas como $(T(t, 0) \phi)(0)=x_{i}(0, \phi)(0)=x(t)$ donde $\phi \in \mathcal{R} P^{+}(0) \cap \mathcal{R} Q^{-}(0)$ para todo $t \in \mathbb{R}$. Portanto:

$$
\mathcal{N}(F)=\left\{(T(t, 0) \phi)(0): \phi \in \mathcal{R} P^{+}(0) \cap \mathcal{R} Q^{-}(0), t \in \mathbf{R}\right\} .
$$

Caracterizemos $\mathcal{R}(F)$.

Seja $y \in C_{b}^{0}\left(\mathbf{R}, \mathbf{R}^{\mathbf{n}}\right)$ solução do sistema adjunto formal em $\mathbf{R}$. Como $\int_{u}^{u+r}|L(\alpha)| d \alpha<M r$ para todo $u \in \mathbf{R}$, o lema 3.4 implica que $\tilde{\Psi}_{t} y \stackrel{\text { def }}{=} \tilde{\Psi}(y)(t)$ é uma trajetória limitada de $T^{*}(s, t)$ em $\mathrm{R}$ logo existe $\eta_{0} \in B_{0}$ tal que $T^{*}(t, 0) \eta_{0}=$ $\tilde{\Psi}_{t}(y)$. Como $T(t, s)$ tem dicotomia exponencial em $[0,+\infty)$ e $(-\infty, 0], T^{*}(s, t)$ também tem com projeções $P^{+*}(t), Q^{+*}(t)$ e $P^{-*}(t) Q^{-*}(t)$, respectivamente. Tomando $t \rightarrow-\infty$ obtemos que $\eta_{0} \in \mathcal{R} P^{-*}(0)$,

$$
\left|\Psi_{t}(y)\right|=\left|T^{*}(t, 0) P^{-*}(0) \eta_{0}\right| \leq K e^{\alpha t}\left|\eta_{0}\right|
$$

$e \eta_{0} \in \mathcal{R} Q^{+*}(0)$

$$
\left|\tilde{\Psi}_{t}(y)\right|=\left|T^{*}(t, 0) Q^{+*}(0) \eta_{0}\right| \leq K e^{-\alpha t}\left|\eta_{0}\right| .
$$

se $t \rightarrow+\infty$.

Concluímos então que

$$
\tilde{\Psi}_{t}(y) \rightarrow 0
$$

exponencialmente se $t \rightarrow \pm \infty$, o que implica,

$$
\psi(t, .) \rightarrow 0
$$

exponencialmente para $t \rightarrow \pm \infty$. Ainda,

$$
y(t)=-\psi\left(t, 0^{-}\right) \rightarrow 0
$$

exponencialmente tomando $t \rightarrow \pm \infty$. Portanto, se $h \in \mathcal{R}(F), \int_{-\infty}^{+\infty} y(t) h(t) d t$ é convergente.Logo, se $\sigma \rightarrow-\infty$ e $t \rightarrow+\infty$ na fórmula do lema 1.5 obtemos que

$$
\int_{-\infty}^{+\infty} y(t) h(t) d t=0
$$

Daí,

$h \in\left\{h: h \in C_{b}^{0}\left(\mathbf{R}, \mathbf{R}^{\mathrm{n}}\right), \int_{-\infty}^{+\infty} y(t) h(t) d t=0\right.$ para todo $y \in C_{b}^{0}\left(\mathbf{R}, \mathbf{R}^{\mathrm{n}}\right)$ satisfazendo o sistema adjunto formal (1.6)\}. 
Consideremos agora $h \in\left\{h: h \in C_{b}^{0}\left(\mathbf{R}, \mathbf{R}^{\mathbf{n}}\right), \int_{-\infty}^{+\infty} y(t) h(t) d t=0\right.$ para todo $y \in C_{b}^{0}\left(\mathbf{R}, \mathbf{R}^{\mathbf{n}}\right)$ satisfazendo o sistema adjunto formal (1.6)\}.

Para que $h \in \mathcal{R}(F)$ devemos mostrar que existe $\phi \in C$ que satisfaz as seguintes equações obtidas nos teoremas 3.1 e 3.2 :

$$
\begin{aligned}
x_{t}= & T(t, 0) P^{+}(0) \phi+\int_{0}^{t} T(t, s) P^{+}(s) X_{0} h(s) d s \\
& -\int_{t}^{\infty} T(t, s) Q^{+}(s) X_{0} h(s) d s, t \geq 0 \\
x_{t}= & T(t, 0) Q^{-}(0) \phi+\int_{0}^{t} T(t, s) Q^{-}(s) X_{0} h(s) d s \\
& +\int_{-\infty}^{t} T(t, s) P^{-}(s) X_{0} h(s) d s, t \leq 0 .
\end{aligned}
$$

Quando $t=0$, obtemos de (3.6) e (3.7):

$$
\begin{aligned}
& x_{0}=P^{+}(0) \phi-\int_{0}^{\infty} T(0, s) Q^{+}(s) X_{0} h(s) d s \\
& x_{0}=Q^{-}(0) \phi+\int_{-\infty}^{\tilde{\omega}} T(0, s) P^{-}(s) X_{0} h(s) d s
\end{aligned}
$$

Logo, (3.6) e (3.7) têm solução $\phi \in C$ se, e somente se,

$$
\begin{aligned}
\left(P^{+}(0)-Q^{-}(0)\right) \phi= & \int_{-\infty}^{0} T(0, s) P^{-}(s) X_{0} h(s) d s \\
& +\int_{0}^{+\infty} T(0, s) Q^{+}(s) X_{0} h(s) d s
\end{aligned}
$$

Como $P^{+}(0)$ é Fredholm $\left(\operatorname{dim} \mathcal{R} Q^{+}(0)\right.$ é finita) e $Q^{-}(0)$ é de imagem finita em $C$ e portanto é compacto segue que $P^{+}(0)-Q^{-}(0)$ é Fredholm: Portanto, $R\left[P^{+}(0)-Q^{-}(0)\right]$ é fechado, o que implica $R\left[P^{+}(0)-Q^{-}(0)\right]={ }^{0}\left[N\left(P^{+}(0)-\right.\right.$ $\left.\left.Q^{-}(0)\right)^{*}\right]$ e então (3.8) possui uma solução $\phi \in C$ se, e só se,

$$
<\psi, \int_{-\infty}^{0}+\int_{0}^{+\infty}>=0
$$

para todo $\psi \in N\left(P^{+}(0)-Q^{-}(0)\right)^{*}=N\left(P^{+*}(0)-Q^{-*}(0)\right)$.

Se $\psi \in N\left(P^{+*}(0)-Q^{-*}(0)\right)$ temos que $\left(P^{+*}(0)-Q^{-*}(0)\right) \psi=0$ ou $\left(I-Q^{+*}(0)-I+P^{-*}(0)\right) \psi=0$ ou

$$
P^{-*}(0) \psi=Q^{+*}(0) \psi \stackrel{\text { def }}{=} \psi_{0} \text { e } \psi_{0} \in \mathcal{R} P^{-*}(0) \cap \mathcal{R} Q^{+*}(0)
$$


A função $\bar{\psi}(t,.) \stackrel{\text { def }}{=} T^{*}(t, 0) \psi_{0}, t \in \mathbf{R}$ é uma trajetória do sistema adjunto em $B_{0}$ e $\bar{\psi}(t,.) \rightarrow 0$ exponencialmente quando $t \rightarrow \pm \infty$. Ainda,

$$
\begin{aligned}
<\psi, \int_{-\infty}^{0}+\int_{0}^{+\infty}>= & \int_{-\infty}^{0}<\psi, T(0, s) P^{-}(s) X_{0}>h(s) d s \\
& +\int_{0}^{\infty}<\psi, T(0, s) Q^{+}(s) X_{0}>h(s) d s \\
= & \int_{-\infty}^{0}<T^{*}(s, 0) P^{-*}(0) \psi, X_{0}>h(s) d s \\
& +\int_{0}^{\infty}<T^{*}(s, 0) Q^{+*}(0) \psi, X_{0}>h(s) d s \\
= & \int_{-\infty}^{0}<T^{*}(s, 0) \psi_{0}, X_{0}>h(s) d s \\
& +\int_{0}^{\infty}<T^{*}(s, 0) \psi_{0}, X_{0}>h(s) d s \\
= & \int_{-\infty}^{+\infty}<T^{*}(s, 0) \psi_{0}, X_{0}>h(s) d s \\
= & -\int_{-\infty}^{+\infty} \bar{\psi}\left(s, 0^{-}\right) h(s) d s .
\end{aligned}
$$

Pelo lema $3.4, \bar{\psi}\left(s, 0^{-}\right), s \in \mathbf{R}$ é uma trajetória limitada do sistema adjunto formal em R. Portanto,

$$
<\psi, \int_{-\infty}^{0}+\int_{0}^{+\infty}>=0
$$

se $\int_{-\infty}^{\infty} y(t) h(t) d t=0$ para todas as trajetórias limitadas do sistema adjunto formal , $y($.$) . A caracterização de \mathcal{R}(F)$ está justificada.

Obtemos ainda que

$$
\begin{gathered}
\operatorname{dim} \mathcal{N}(F)=\operatorname{dim}\left(\mathcal{R} P^{+}(0) \cap \mathcal{R} Q^{-}(0)\right) \\
\operatorname{dim}\left(C_{b}^{0} / \mathcal{R}(F)\right)=\operatorname{codim} \mathcal{R}(F)=\operatorname{dim}\left(\mathcal{R} P^{-*}(0) \cap \mathcal{R} Q^{+*}(0)\right),
\end{gathered}
$$

e ambas são finitas. Portanto, $F$ é Fredholm e

$$
I(F)=\operatorname{dim}\left[\mathcal{R} P^{+}(0) \cap \mathcal{R} Q^{-}(0)\right]-\operatorname{dim}\left[\mathcal{R} P^{-*}(0) \cap \mathcal{R} Q^{+*}(0) .\right.
$$

Além disso, $\mathcal{R}(F)$ é fechado em $C_{b}^{0}\left(\mathbf{R}, \mathbf{R}^{\mathbf{n}}\right)$ e $\mathcal{N}(F)$ é fechado em $C_{b}^{\mathbf{1}}\left(\mathbf{R}, \mathbf{R}^{\mathbf{n}}\right)$.

Mostremos que $I(F)=\operatorname{dim} \mathcal{R} Q^{-}(0)-\operatorname{dim} \mathcal{R} Q^{+}(0)$.

Seja $\left\{q_{1}^{-}, q_{2}^{-}, \ldots, q_{k}^{-}\right\}$uma base de $\mathcal{R} Q^{-}(0) \cap \mathcal{R} P^{+}(0)$. Podemos acrescentar $\left\{q_{k+1}^{-}, \ldots, q_{m_{1}}^{-}\right\}$, onde $m_{1}=\operatorname{dim} \mathcal{R} Q^{-}(0)$ para formar a base $\left\{q_{1}^{-}, \ldots, q_{m_{1}}^{-}\right\}$em 
$\mathcal{R} Q^{-}(0)$. Também escolhemos uma base $\left\{q_{1}^{+*}, \ldots, q_{h}^{+*}\right\}$ em $\mathcal{R} Q^{+*}(0) \cap \mathcal{R} P^{-*}(0)$ e adicionamos $\left\{q_{h+1}^{+*}, \ldots, q_{m_{2}}^{+*}\right\}$ para formar uma base $\left\{q_{1}^{+*}, \ldots, q_{m_{2}}^{+*}\right\}$ em $\mathcal{R} Q^{+*}(0)$, onde $m_{2}=\operatorname{dim} \mathcal{R} Q^{+*}(0)$.

Seja $\left[e_{1}, \ldots, e_{\mu}\right]$ o espaço gerado pelos velores $\left\{e_{1}, \ldots, e_{\mu}\right\}$. Se $q^{*} \neq 0, q^{*} \in$ $\left[q_{h+1}^{+*}, \ldots, q_{m_{2}}^{+*}\right]$, afirmamos que existe $q \in\left[q_{k+1}^{-}, \ldots, q_{m_{1}}^{-}\right]$tal que $<q^{*}, q>\neq 0$. Caso contrário, $q^{*} \in\left[q_{k+1}^{-}, \ldots, q_{m_{1}}^{-}\right]^{0}$ e já sabemos que $q^{*} \in$ $\left[q_{1}^{-}, \ldots, q_{k}^{-}\right]^{0} ;$ pois para $q_{i}^{-} ; i=1,2, \ldots, k$

$$
<q^{*}, q_{i}^{-}>=<q^{*}, P^{+}(0) q_{i}^{-}>=<P^{+*}(0) q^{*}, q_{i}^{-}>=0 .
$$

Então, $q^{*} \in\left[\mathcal{R} Q^{-}(0)\right]^{0}=\mathcal{R} P^{-*}(0)$ e $q^{*} \in \mathcal{R} P^{-*}(0) \cap \mathcal{R} Q^{+*}(0)=\left[q_{1}^{+*}, \ldots, q_{h}^{+*}\right]$, contradizendo o fato que $\left[q_{1}^{+*}, \ldots, q_{h}^{+*}\right] \cap\left[q_{h+1}^{+*}, \ldots, q_{m_{2}}^{+*}\right]=\{0\}$.

Usando o mesmo tipo de argumento mostra-se que, para $q \neq 0$, $q \in\left[q_{k+1}^{-}, \ldots, q_{m_{1}}^{-}\right]$, existe um $q^{*} \in\left[q_{h+1}^{+*}, \ldots, q_{m_{2}}^{+*}\right]$ tal que $\left\langle q^{*}, q>\neq 0\right.$.

Portanto $\operatorname{dim}\left[q_{k+1}^{-}, \ldots, q_{m_{1}}^{-}\right]=\operatorname{dim}\left[q_{h+1}^{+*}, \ldots, q_{m_{2}}^{+*}\right]$, isto é, $m_{1}-k=m_{2}-h$. Finalmente,

$$
I(F)=\operatorname{dim} \mathcal{R} Q^{-}(0)-\operatorname{dim} \mathcal{R} Q^{+}(0) . \square
$$




\section{Capítulo 4}

\section{Bifurcação de Órbitas Homoclínicas}

Neste capítulo estudamos condições necessárias e suficientes para que uma equação que é uma perturbação não autônoma periódica de uma equação dife-

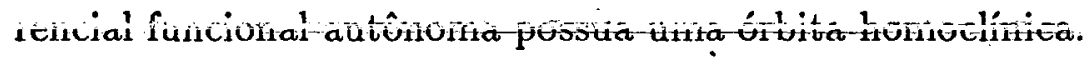

Consideramos a equação escalar retardada:

$$
\dot{x}(t)=\alpha(x(t)) x(t)+\beta(x(t)) x(t-1)
$$

onde:

- $\alpha(x), \beta(x)$ são funções escalares $C^{\infty}$. definidas em $\mathbf{R}$.

- $x \equiv 0$ é um ponto de equilíbrio hiperbólico de

$$
\dot{x}(t)=\alpha(0) x(t)+\beta(0) x(t-1)
$$

$\operatorname{com} \operatorname{dim} W^{u}(0)=1$ e $\operatorname{codim} W^{s}(0)=1$. $W^{u}(0)$ e $W^{s}(0)$ são respectivamente, os subespaços instável e estável associados a $x=0$.

- $x \stackrel{\text { def }}{=} p(t), t \in \mathbf{R}$, é uma órbita (limitada) em $\mathbf{R}$ de (4.1) tal que $p(t) \rightarrow 0$ assintoticamente quando $t \rightarrow \pm \infty$, isto é, uma trajetória homoclínica assintótica a $x \equiv 0$ quando $t \rightarrow \pm \infty$.

- existe $\eta>0$ tal que $-\beta(x) \geq \eta$ para $x \in[-k, k]$ onde $k=\operatorname{máx}\{p(t)$ : $t \in \mathbf{R}\}$. 
Estudaremos o comportamento das soluções homoclínicas perto de $x=p(t)$ para o sistema perturbado de (4.1):

$$
\dot{x}(t)=\alpha(x(t)) x(t)+\beta(x(t)) x(t-1)+h\left(t, x_{t}, \mu\right)
$$

onde:

- $\mu \in X, X$ espaço de Banach.

- $h: \mathbf{R} \times C \times X \rightarrow \mathbf{R}$ é $C^{1}$ com derivada limitada; $h(t+w, \phi, \mu)=$ $h(t, \phi, \mu), w>0$, uma constante; $h(t, \phi, \mu)=O(|\mu|)$ para $\mu$ próximo de zero uniformemente com respeito a $t, \phi$. Observe que as condições acima implicain que $h(t, \phi, 0)=0$ e reciprocamente.

Utilizaremos as técnicas de Liapunov-Schmidt e a Alternativa de Fredholm.

Definição 4.1 Se $p(t)$ é uma solução, não constante, da equaçâao

$$
\dot{x}(t)=f\left(x_{t}\right)
$$

a equacão variacional linear relativa a $p$ é definida como:

$$
\dot{y}(t)=L(t) y_{t}
$$

onde $L(t) \dot{\phi}=\dot{f}\left(p_{t}\right), \dot{f}(\psi)$ é a derivada de Fréchet de $f$ em $\psi$.

Observação 4.1 Se $\dot{p}(t)=f\left(p_{t}\right)$ para $-\infty<t<+\infty$ segue que $\ddot{p}(t)=\dot{f}\left(p_{t}\right) \dot{p}_{t}$, o que implica, $\dot{p}(t)$ é uma solução de $\dot{y}(t)=L(t) y_{t}$.

\section{Como:}

$$
f(\phi)=\alpha(\phi(0)) \phi(0)+\beta(\phi(0)) \phi(-1)
$$

Derivando em relação a $\phi$ obtemos:

$$
\begin{aligned}
\frac{\partial f\left(p_{t}\right)}{\partial \phi} \phi= & \left.\frac{d}{d \lambda}\left[f\left(p_{t}+\lambda \phi\right)\right]\right|_{\lambda=0} \\
= & {[\dot{\alpha}(p(t)) p(t)+\alpha(p(t))+\dot{\beta}(p(t)) p(t-1)] \phi(0) } \\
& +\beta(p(t)) \phi(-1)
\end{aligned}
$$

Portanto a equação variacional linear de (4.1) com respeito a $x=p(t)$ será

$$
\begin{aligned}
\dot{x}(t) & =\mathcal{A}(t) x(t)+\mathcal{B}(t) x(t-1) \\
\mathcal{A}(t) & =\alpha(p(t))+\dot{\alpha}(p(t)) p(t)+\dot{\beta}(p(t)) p(t-1), \\
\mathcal{B}(t) & =\beta(p(t))
\end{aligned}
$$


e sua adjunta formal

$$
\dot{y}(t)=-\mathcal{A}(t) y(t)-\mathcal{B}(t+1) y(t+1) .
$$

Seja

$$
\begin{aligned}
L(t): C & \rightarrow \mathbf{R} \\
L(t) \phi & =\mathcal{A}(t) \phi(0)+\mathcal{B}(t) \phi(-1)
\end{aligned}
$$

Lema 4.1 $O$ operador $L(t)$ é contínuo com respeito a $t \in \mathbf{R}$ e é uniformemente limitado.

Demonstração Basta observar que $t \mapsto \mathcal{A}(t), t \mapsto \mathcal{B}(t)$ são funções contínuas e que $\mathcal{A}(t) \rightarrow \alpha(0), \mathcal{B}(t) \rightarrow \beta(0)$ quando $t \rightarrow \pm \infty$. $\square$

Lema 4.2 Consideremos o operador solução $T(t, s)$ de (4.3). Então:

(i) Para cada $0 \neq \phi \in C, T(t, s) \phi \neq 0, t \geq s$, isto é, $T(t, s)$ é injetor.

(ii) Para cada $0 \neq \psi \in B_{0}, \tilde{T}(s, t) \psi \neq 0, s \leq t$.

Demonstraçäo Como $-\hat{\beta}(x) \geq \eta>\dot{0}$ para $x \in[-\dot{k}, \dot{k} j$ obtemos que $-\mathcal{B}(t) \geq \eta>0$ para todo $t \in \mathbf{R}$.

Sabemos que

$$
L(t) \phi=\dot{A}(t) \phi(0)+\mathcal{B}(t) \phi(-1)
$$

Por outro lado:

$$
L(t) \phi=\int_{-1}^{0} d_{\theta} \eta(t, \theta) \phi(\theta)
$$

logo,

$$
\eta(t, \theta)= \begin{cases}-\mathcal{A}(t)-\mathcal{B}(t) & \theta \leq-1 \\ -\mathcal{A}(t) & \theta \in(-1,0) \\ 0 & \theta \geq 0\end{cases}
$$

E, portanto,

$$
A(t,-1)=\eta\left(t,-1^{+}\right)-\eta\left(t,-1^{-}\right)=\mathcal{B}(t)<0
$$

- que implica, $L(t) \phi$ atômica em -1 .

(i) Segue do lema 1.2.

(ii) Com argumentos similares aos utilizados em (i) concluímos que para todo $\psi \in B_{0}, \psi \neq 0, \tilde{T}(s, t) \psi \neq 0$ para $s \leq t . \square$ 
Lema 4.3 A equação (4.3) possui uma dicotomia exponencial em $\mathbf{R}_{+}$e $\mathbf{R}_{-}$com $\operatorname{dim} \mathcal{R} Q^{-}(0)=1=\operatorname{dim} \mathcal{R} Q^{+}(0) . F=\frac{d}{d t}-L(t)$ é um operador de Fredholm de $C_{b}^{1}(\mathbf{R})$ em $C_{b}^{0}(\mathbf{R}) \operatorname{com} I(F)=0$.

Demonstração Pelo lema anterior e dos fatos que $\mathcal{A}(t) \rightarrow \alpha(0), \mathcal{B}(t) \rightarrow \beta(0)$ quando $t \rightarrow \pm \infty$ e $\dot{x}(t)=\alpha(0) x(t)+\beta(0) x(t-1)$ possui o zero como um ponto de equilíbrio hiperbólico concluímos do teorema 3.3 que (4.3) possui dicotomia exponencial em $\mathbf{R}_{+}$e $\mathbf{R}_{-} \operatorname{com} \operatorname{dim} \mathcal{R} Q^{ \pm}(t)=1$. Do teorema 3.4 obtemos então que $F=\frac{d}{d t}-L(t)$ é um operador de Fredholm de $C_{b}^{1}(\mathbf{R})$ em $C_{b}^{0}(\mathbf{R})$ e $I(F)=0 . \square$

Da demonstração do teorema 3.4 sabemos que:

$$
\operatorname{dim} \mathcal{N}(F)=\operatorname{dim}\left[\mathcal{R} P^{+}(0) \cap \mathcal{R} Q^{-}(0)\right] \leq 1 .
$$

Mas $\dot{p}(t) \in \mathcal{N}(F)$ o que implica $\operatorname{dim} \mathcal{N}(F)=1$ e $\mathcal{N}(F)=[\dot{p}(t)]$. Como $I(F)=0$; $\operatorname{codim} \mathcal{R}(F)=1$. Logo, (4.4) possui uma única solução limilada $y=y_{0}(t) \neq 0$, $t \in \mathbf{R}$, a menos da multiplicação por um escalar e que $y_{0}(t) \rightarrow 0$ exponencialmente quando $t \rightarrow \pm \infty$. $\mathrm{E}$

$$
\mathcal{K}(F)=\left\{h \in C_{b}^{\hat{0}}(K): \int_{-\infty}^{+\infty} y_{0}(t) h(t) d t=u\right\}
$$

Assumimos que $\int_{-\infty}^{+\infty} y_{0}^{2}(t) d t=1$ e escolhemos $t_{0} \in \mathbf{R}$ tal que $\dot{p}\left(t_{0}\right) \neq 0$. Definimos: $x_{0}(t)=\dot{p}(t) / \dot{p}\left(t_{0}\right)$. Assim, $x_{0}(t)$ gera $\mathcal{N}(F)$ e $x_{0}\left(t_{0}\right)=1$. Temos então a definição:

Definição 4.2 Consideremos as projeções $E_{1}$ e $E_{2}$ definidas por:

$$
\begin{aligned}
E_{1}: C_{b}^{1}(\mathbf{R}) & \rightarrow \mathcal{N}(F) \\
x & \mapsto E_{1}(x)(t)=x\left(t_{0}\right) x_{0}(t) \\
E_{2}: C_{b}^{0}(\mathbf{R}) & \rightarrow \mathcal{R}(F) \\
f & \mapsto E_{2}(f)(t)=f(t)-y_{0}(t) \int_{-\infty}^{+\infty} y_{0}(s) f(s) d s
\end{aligned}
$$

Lema 4.4 Nas condiçóes acima temos:

(i) $x \in \mathcal{N}\left(E_{1}\right)$ se, e somente se, $x \in C_{b}^{1}(R)$ e $x\left(t_{0}\right)=0$.

(ii) $f \in \mathcal{N}\left(E_{2}\right)$ se, e somente se $f=k y_{0}$ para alguma constante $k$.

Demonstração Imediata. 
Lema 4.5 A aplicação

$$
\begin{aligned}
\left.F\right|_{\mathcal{N}\left(E_{1}\right)}: \mathcal{N}\left(E_{1}\right) & \rightarrow \mathcal{R}\left(E_{2}\right) \\
x & \mapsto\left(\left.F\right|_{\mathcal{N}\left(E_{1}\right)}\right)(x)(t)=\dot{x}(t)-\mathcal{A}(t) x(t)-\mathcal{B}(t) x(t-1)
\end{aligned}
$$

é um isomorfismo cuja inversa é a função

$$
\mathcal{K}: \mathcal{R}\left(E_{2}\right) \rightarrow \mathcal{N}\left(E_{1}\right)
$$

tal que

$$
|\mathcal{K} f|_{C_{b}^{1}} \leq M|f|_{C_{b}^{0}}
$$

onde $M$ é uma constante positiva e $f \in \mathcal{R}\left(E_{2}\right)=\mathcal{R}(F)$.

Demonstração Da definição de $E_{1}$ e $E_{2}$ segue que $\left.F\right|_{\mathcal{N}\left(E_{1}\right)}$ é um isomorfismo. Decorre do teorema da Aplicação Aberta que existe $M>0$ tal que

$$
|\mathcal{K} f|_{C_{b}^{1}} \leq M|f|_{C_{b}^{0}}
$$

para todo $t \in \mathbf{R} . \square$

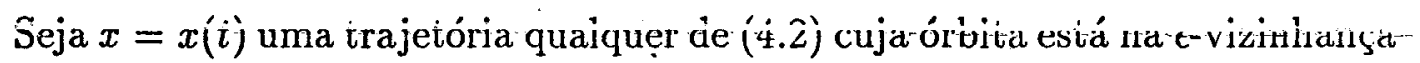
da órbita de $x=p(t)$, ambas em $C[-1,0]$. Escrevemos então

$$
x(\tau)=p(\tau+\zeta)+z(\tau+\zeta)
$$

Como $\dot{p}\left(t_{0}\right) \neq 0$, podemos escolher $\zeta$ tal que $z\left(t_{0}\right)=0$, se $\epsilon$ é suficientemente pequeno. Obtemos então que

$$
z \in \mathcal{R}\left(I-E_{1}\right)
$$

Como

$$
F(z)(t)=\dot{z}(t)-\mathcal{A}(t) z(t)-\mathcal{B}(t) z(t-1)
$$

encontrar uma solução $x$ de (4.2) é equivalente a encontrar uma solução $z$ de

$$
F(z)(t)=f(z, \zeta, \mu)(t)
$$

onde $f: C_{b}^{0}(\mathbf{R}) \times \mathbf{R} \times X \rightarrow C_{b}^{0}(\mathbf{R})$ é definida por

$$
\begin{aligned}
f(z, \zeta, \mu)= & \alpha(p(t)+z(t))(p(t)+z(t)) \\
& +\beta(p(t)+z(t))(p(t-1)+z(t-1)) \\
& -[\alpha(p(t)) p(t)+\beta(p(t)) p(t-1)] \\
& -[\mathcal{A}(t) z(t)+\mathcal{B}(t) z(t-1)] \\
& +-h\left(t-\zeta, p_{t}+z_{t}, \mu\right)
\end{aligned}
$$


Como $h(t, \phi, \mu)=O(|\mu|)$ para $\mu$ perto do zero uniformemente em $t, \phi$, temos que:

(1) $f(0, \zeta, 0)(t)=h\left(t-\zeta, p_{t}, 0\right)=0$,

(2) $f_{z}(0, \zeta, 0)(z)(t)=\left.\left[\frac{d}{d \lambda} f(\lambda z, \zeta, 0)(t)\right]\right|_{\lambda=0}=0$

(3) Como $f(0, \zeta, 0)=0, f_{z}(0 . \zeta, 0)=0$ e $f_{\mu}(0, \zeta, 0)=h_{\mu}\left(t-\zeta, p_{t}, 0\right)=O(|\mu|)$, $f(z, \zeta, \mu)=O(|\mu|)+o\left(\sqrt{|z|^{2}+|\mu|^{2}}\right)$. Para $(z, \mu)$ arbitrariamente próximos de $(0,0)$, obtemos então que:

$$
f(z, \zeta, \mu)=O\left(|z|^{2}+|\mu|\right) .
$$

Pelo método de Liapunov - Schmidt (4.6) é equivalente a

$$
\begin{aligned}
F(z) & =E_{2} f(z, \zeta, \mu) \\
0 & =\left(I-E_{?}\right) f(z, \zeta, \mu)
\end{aligned}
$$

Por (4.5), a primeira igualdade de (4.7) é equivalente a

$$
\boldsymbol{z}=\mathcal{K} E_{2} f(z, \zeta, \mu)
$$

Visto que $f: C_{b}^{0}(\mathbf{R}) \times \mathbf{R} \times X \rightarrow C_{b}^{0}(\mathbf{R})$ é de classe $C^{1}$ numa vizinhança de $(0, \zeta, 0) \operatorname{com} f(0, \zeta, 0)=0$ e $f_{z}(0, \zeta, 0)=0$, então a função

$$
\begin{aligned}
g: C_{b}^{1}(\mathbf{R}) \times \mathbf{R} \times X & \rightarrow C_{b}^{1}(\mathbf{R}) . \\
g(z, \zeta, \mu) & =z-\mathcal{X} E_{2} f(z, \zeta, \mu)
\end{aligned}
$$

tem as seguintes propriedades:

$$
\begin{array}{r}
g(0, \zeta, 0)=0 \\
\frac{\partial g}{\partial z}(0, \zeta, 0)=I
\end{array}
$$

Pelo teorema da Função Implícita segue que existem bolas abertas $B_{C_{b}^{1}}\left(\epsilon_{1}\right) \subset$ $C_{b}^{1}, B_{X}\left(\epsilon_{2}\right) \subset X$ com raio $\epsilon_{1}, \epsilon_{2}$ ambas com centros em zero onde a equação $g(z, \zeta, \mu)=0$ possui uma única solução $C^{1}, z=\dot{z}^{*}(\zeta, \mu)$

$$
z^{*}(\zeta, \mu): R \times B_{X}\left(\epsilon_{2}\right) \rightarrow B_{C_{b}^{1}}\left(\epsilon_{1}\right)
$$


Como $z^{*}(\zeta, 0)=0, f_{z}(0, \zeta, 0)=0$ e $f_{\mu}(0, \zeta, 0) \mu=O(|\mu|)$ segue que $z^{*}(\zeta, \mu)=$ $O(|\mu|)$ para $\mu$ próximo do zero, uniformemente com respeito a $\zeta \in \mathbb{R}$.

A equação de bifurcação é obtida pela substituição de $z^{*}(\zeta, \mu)$ na segunda. equação de (4.7)

$$
G(\zeta, \mu) \stackrel{\text { def }}{=} \int_{-\infty}^{+\infty} y_{0}(t) f\left(z^{*}(\zeta, \mu), \zeta, \mu\right)(t) d t=0
$$

e, portanto

$$
G(\zeta, \mu)=\int_{-\infty}^{+\infty} y_{0}(t) h\left(t-\zeta, p_{t}, \mu\right) d t+o(|\mu|) .
$$

pois $f_{z}(0, \zeta, 0)=0$ e $f_{\mu}(0, \zeta, 0)(t) \mu=h_{\mu}\left(t-\zeta, p_{t}, 0\right) \mu=h\left(t-\zeta, p_{t}, \mu\right)-o(|\mu|)$.

Como

$$
\int_{-\infty}^{+\infty} y_{0}(t)[g(t)] d t: C_{b}^{0}(\mathbf{R}) \rightarrow \mathbf{R}
$$

é um funcional linear contínuo,

$$
G(\zeta, \mu): \mathbf{R} \times X \rightarrow \mathbf{R}
$$

$\dot{\mathrm{e}} C^{\mathbf{1}}$.

Demonstramos então o seguinte teorema:

Teorema 4.1 Consideremos a função $h: \mathbf{R} \times C \times X \rightarrow \mathbf{R}$ de classe $C^{1}$ com derivada limitada; $h(t+w, \phi, \mu)=h(t, \phi, \mu), w>0$, uma constante $e$ $h(t, \phi, 0)=0$.

Então $x=x(t)$ é uma trajetória homoclínica de (4.2), cuja órbita está na vizinhança da órbita de $x=p(t)$ se, e somente se, $x(t)=p(t+\zeta)+z^{*}(\zeta, \mu)(t+\zeta)$ $\operatorname{com} z^{*}(\zeta, \mu)\left(t_{0}\right)=0$ e $G(\zeta, \mu)=0$.

Corolário 4.1 Seja $\mu \in X=\mathbf{R}$. Consideremos que $h: \mathbf{R} \times C \times \mathbf{R} \rightarrow \mathbf{R}$ é de classe $C^{2}$ com todas as derivadas até as de ondem dois limitadas. Seja

$$
\Delta(\zeta) \stackrel{\text { def }}{=} \int_{-\infty}^{+\infty} y_{0}(t) h_{1}\left(t-\zeta, p_{t}, 0\right) d t
$$

com

$$
\begin{aligned}
& h_{1}(t, \phi, \mu)=\frac{1}{\mu} h(t, \phi, \mu), \quad \mu \neq 0 \\
& h_{1}(t, \phi, 0)=\frac{\partial h}{\partial \mu}(t, \phi, 0)
\end{aligned}
$$


Se $\Delta\left(\zeta_{0}\right)=0$ e $\dot{\Delta}\left(\zeta_{0}\right) \neq 0$ para algum $\zeta_{0} \in \mathbf{R}$, então existe uma única trajetória homoclínica de (4.2) perto de $x=p(t)$ para cada $\mu,|\mu| \leq \mu_{0}$. Além disso, $\zeta$ é uma função $C^{1}$ de $\mu, \zeta=\zeta^{*}(\mu),|\mu| \leq \mu_{0}$ e $\zeta_{0}=\zeta^{*}(0)$, e a trajetória homoclínica é dada por:

$$
x(t)=p\left(t+\zeta^{*}(\mu)\right)+z^{*}\left(\mu, \zeta^{*}(\mu)\right)\left(t+\zeta^{*}(\mu)\right) .
$$

Demonstração Use o teorema da Função Implícita para a função $H: \mathbf{R} \times \mathbf{R} \rightarrow \mathbf{R}$ definida por:

$$
\begin{aligned}
H(\zeta, \mu) & =\frac{1}{\mu}\left[\int_{-\infty}^{+\infty} y_{0}(t) h\left(t-\zeta, p_{t}, \mu\right) d t+o(|\mu|)\right], \quad \mu \neq 0 \\
H(\zeta, 0) & =\int_{-\infty}^{+\infty} y_{0}(t) \frac{\partial h}{\partial \mu}\left(t-\zeta, p_{t}, 0\right) d t .
\end{aligned}
$$

Um caso especial em que as condições do corolário 4.1 sã̃o facilmente verifiradas é apresentada no cornlário ahaixo.

Corolário 4.2 Seja $h(t, \phi, \mu)=\mu \cos t, \mu \in \mathbf{R}$. Definimos

$$
\Delta(\zeta) \stackrel{\text { def }}{=} \int_{-\infty}^{+\infty} y_{0}(t) \cos (t-\zeta) d t
$$

Se $\Delta(\zeta) \not \equiv 0$, então as condições do corolário 4.1 são satisfeitas para algum $\zeta_{0}$.

Exemplo: Considere a equação escalar retardada

$$
\dot{x}(t)=(\cosh 1)(\sinh 1)^{-1} x(t)-(\sinh 1)^{-1}\left(1+x^{2}(t)\right) x(t-1)+\mu \cos t
$$

onde $\mu \in \mathbf{R}$. Se $\mu=0, x(t)=(\sinh 1)(\cosh t)^{-1}$ é solução homoclínica da equação autônoma. 


\section{Bibliografia}

[1] BANKS H. T. The representation of solutions of linear functional differential equations, J. Differential Equations v.5, p. 399-410, 1969.

[2] BACHMAN G.; NARICI L. Functional Analysis. New York, Academic Press, 1966.

[3] CAMERON R. H.; MARTIN W. T. An unsymmetric Fubini theorem. Bull. Amer. Math. Soc., v. 47, p. 121-5, 1941

[4] CARVAlHo A: N. Dicotomia Discreta e Aplicações. São Carlos, ICMSC - USP, 1987. (Dissertação de Mestrado)

[5] HALE J. K. Introduction on Dynamycal Bifurcation. LCDS Report n.83$16,1983$.

[6] HALE J. K. Theory of Functional Differential Equations. New York, Springer, 1977.

[7] LIN X. B. Exponential dichotomies and homoclinic orbits in functional differential equations. J. Differential Equations, v.63, p. 227-54, 1986.

[8] PALMER K. J. Exponential dichotomies and transversal homoclinic points. J. Differential Equations, v.55, p. 225-56, 1984.

[9] RUAS V. Introdução aos Problemas Variacionais. Rio de Janeiro, Guanabara Dois, 1979.

[10] TAYLOR A. E.; LAY D. C. Introduction to Functional Analysis, 2 ed., New York, John Wiley, 1980.

is 